Pacific

Journal of

Mathematics

\title{
HARMONIC MAPS ON DOMAINS WITH PIECEWISE LIPSCHITZ CONTINUOUS METRICS
}

HAIGANG Li AND CHANGYOU WANG 


\title{
HARMONIC MAPS ON DOMAINS WITH PIECEWISE LIPSCHITZ CONTINUOUS METRICS
}

\author{
HAIGANG Li AND CHANGYOU WANG
}

\begin{abstract}
We study harmonic maps $(\Omega, g) \rightarrow(N, h)$, where $\Omega \subset \mathbb{R}^{n}$ is a bounded domain divided into two pieces, the Riemannian metric $g$ is Lipschitz in each piece, and $(N, h)$ is a closed Riemannian submanifold of $\mathbb{R}^{k}$. We prove the partial regularity of stationary harmonic maps, and the global Lipschitz and piecewise $C^{1, \alpha}$-regularity of weakly harmonic maps from $(\Omega, g)$ to manifolds $(N, h)$ that support convex distance square functions.
\end{abstract}

\section{Introduction}

Throughout this paper we assume that $\Omega=\Omega^{+} \cup \Omega^{-} \cup \Gamma$ is a bounded domain of $\mathbb{R}^{n}$ decomposed into two subdomains $\Omega^{+}$and $\Omega^{-}$by a $C^{1,1}$-hypersurface $\Gamma$, and that $g$ is a piecewise Lipschitz metric on $\Omega$, satisfying $g \in C^{0,1}\left(\Omega^{+}\right) \cap C^{0,1}\left(\Omega^{-}\right)$ and discontinuous at every $x \in \Gamma$. For example, let $\Omega=B_{1} \subset \mathbb{R}^{n}$ be the unit ball, $\Gamma=B_{1} \cap\left\{x=\left(x^{\prime}, 0\right) \in \mathbb{R}^{n}\right\}$, and

$$
\bar{g}(x)= \begin{cases}g_{0} & \text { if } x \in B_{1}^{+}=\left\{x^{n}>0\right\} \cap B_{1}, \\ k g_{0} & \text { if } x \in B_{1}^{-}=\left\{x^{n}<0\right\} \cap B_{1},\end{cases}
$$

where $g_{0}$ is the standard metric on $\mathbb{R}^{n}$ and $k(\neq 1)$ is a positive constant. Let $(N, h) \hookrightarrow \mathbb{R}^{k}$ be an $l$-dimensional, smooth compact Riemannian manifold without boundary, isometrically embedded in the Euclidean space $\mathbb{R}^{k}$.

Motivated by the recent studies on elliptic systems arising from composite materials (see [ $\mathrm{Li}$ and Nirenberg 2003]) and the periodic homogenization theory in calculus of variations (see [Avellaneda and Lin 1987] and [Lin and Yan 2003]), we are interested in the regularity issue of harmonic maps from $(\Omega, g)$ to $(N, h)$.

In order to describe the problem, let's recall some notations. Throughout this paper, we use the Einstein convention for summation. For the metric $g=g_{i j} d x^{i} d x^{j}$, let $\left(g^{i j}\right)=\left(g_{i j}\right)^{-1}$, and $d v_{g}=\sqrt{g} d x\left(=\sqrt{\operatorname{det}\left(g_{i j}\right)} d x\right)$ be the volume form of $g$. For $1<p<+\infty$, define the Sobolev space

$$
W^{1, p}(\Omega, N)=\left\{u: \Omega \rightarrow \mathbb{R}^{k} \mid u(x) \in N \text { a.e. } x \in \Omega, E_{p}(u, g)=\int_{\Omega}|\nabla u|_{g}^{p} d v_{g}<\infty\right\},
$$

Keywords: piecewise continuous metric, harmonic map, regularity. 
where

$$
|\nabla u|_{g}^{2} \equiv g^{i j}\left\langle\frac{\partial u}{\partial x_{i}}, \frac{\partial u}{\partial x_{j}}\right\rangle
$$

is the energy density of $u$ with respect to $g$, and $\langle\cdot, \cdot\rangle$ denotes the inner product in $\mathbb{R}^{k}$. Denote $W^{1,2}(\Omega, N)$ by $H^{1}(\Omega, N)$. Now let's recall the definition of stationary harmonic maps.

Definition 1.1. A map $u \in H^{1}(\Omega, N)$ is called a (weakly) harmonic map if it is a critical point of $E_{2}(\cdot, g)$, i.e., if $u$ satisfies

$$
\Delta_{g} u+A(u)(\nabla u, \nabla u)_{g}=0 \quad \text { in } \Omega
$$

in the sense of distributions. Here

$$
\Delta_{g}=\frac{1}{\sqrt{g}} \frac{\partial}{\partial x_{i}}\left(\sqrt{g} g^{i j} \frac{\partial}{\partial x_{j}}\right)
$$

is the Laplace-Beltrami operator on $(\Omega, g), A(\cdot)(\cdot, \cdot)$ is the second fundamental form of $(N, h) \hookrightarrow \mathbb{R}^{k}$, and $A(u)(\nabla u, \nabla u)_{g}=g^{i j} A(u)\left(\partial u / \partial x_{i}, \partial u / \partial x_{j}\right)$.

Definition 1.2. A (weakly) harmonic map $u \in H^{1}(\Omega, N)$ is called a stationary harmonic map if, in addition, it is a critical point of $E_{2}(\cdot, g)$ with respect to the following domain variations:

$$
\left.\frac{d}{d t}\right|_{t=0} \int_{\Omega}\left|\nabla u^{t}\right|_{g}^{2} d v_{g}=0, \quad \text { with } u^{t}(x)=u\left(F_{t}(x)\right),
$$

where $F(t, x):=F_{t}(x) \in C^{1}\left([-\delta, \delta], C^{1}(\Omega, \Omega)\right)$, for some small $\delta>0$, is a family of diffeomorphisms that satisfies

$$
\begin{cases}F_{0}(x)=x & \text { for } x \in \Omega, \\ F_{t}(x)=x & \text { for }(x, t) \in \partial \Omega \times[-\delta, \delta], \\ F_{t}\left(\overline{\Omega^{ \pm}}\right) \subset \overline{\Omega^{ \pm}} & \text {for } t \in[-\delta, \delta] .\end{cases}
$$

In particular, $F_{t}(\Gamma) \subset \Gamma$ for $0 \leq t \leq \delta$.

It is readily seen that any minimizing harmonic map from $(\Omega, g)$ to $(N, h)$ is a stationary harmonic map. Definition 1.2 implies that a stationary harmonic map on $(\Omega, g)$ is a stationary harmonic map on $\left(\Omega^{ \pm}, g\right)$. Since $g \in C^{0,1}\left(\Omega^{ \pm}\right)$, we can see that $u$ satisfies an energy monotonicity inequality on $\Omega^{ \pm}$. We will show in Section 2 that a stationary harmonic map on $(\Omega, g)$ also satisfies an energy monotonicity inequality in $\Gamma$ under the condition (1-4) below.

The first result is concerned with the (partial) Lipschitz and (partial) piecewise $C^{1, \alpha}$-regularity of stationary harmonic maps. In this context, we are able to extend the well-known partial regularity theorem of stationary harmonic maps on domains 
with smooth metrics, due to Hélein [2002], Evans [1991], and Bethuel [1993]. More precisely:

Theorem 1.1. Let $u \in H^{1}(\Omega, N)$ be a stationary harmonic map on $(\Omega, g)$. Suppose that $g$ satisfies the following jump condition on $\Gamma$ for $n \geq 3$ : for any $x \in \Gamma$, there exists a positive constant $k(x) \neq 1$ such that

$$
\lim _{\substack{y \in \Omega^{+} \\ y \rightarrow x}} g(y)=k(x) \lim _{\substack{y \in \Omega^{-} \\ y \rightarrow x}} g(y) .
$$

There exists a closed set $\Sigma \subset \Omega$, with $H^{n-2}(\Sigma)=0$, such that $u \in \operatorname{Lip}_{\text {loc }}(\Omega \backslash \Sigma, N)$, and for some $0<\alpha<1, u \in C_{\mathrm{loc}}^{1, \alpha}\left(\left(\Omega^{+} \cup \Gamma\right) \backslash \Sigma, N\right) \cap C_{\mathrm{loc}}^{1, \alpha}\left(\left(\Omega^{-} \cup \Gamma\right) \backslash \Sigma, N\right)$.

The jump condition is needed for both energy monotonicity inequalities for $u$ and the piecewise $C^{1, \alpha}$-regularity of $u$.

We point out that in dimension $n=2$, since the energy monotonicity inequality automatically holds for $H^{1}$-maps, Theorem 1.1 holds for any weakly harmonic map from domains of piecewise $C^{0,1}$-metrics, i.e., any weakly harmonic map on domains with piecewise Lipschitz continuous metrics satisfying (1-4) is both Lipschitz continuous and piecewise $C^{1, \alpha}$ for some $0<\alpha<1$.

Weakly harmonic maps from domains with smooth metrics into Riemannian manifolds may not enjoy partial regularity properties in dimensions $n \geq 3$; see [Rivière 1995]. Here we consider weakly harmonic maps on domains with piecewise Lipschitz continuous metrics into a Riemannian manifold $(N, h)$, on which $d_{N}^{2}(\cdot, p)$ is convex for $p \in N$. Such Riemannian manifolds $N$ include those with nonpositive sectional curvatures and geodesic convex balls in Riemannian manifolds. In particular, we extend the classical regularity theorems on harmonic maps on domains with smooth metrics, due to [Eells and Sampson 1964] and [Hildebrandt et al. 1977].

Theorem 1.2. Let $g$ satisfy the conditions of Theorem 1.1. Assume that on the universal cover $(\tilde{N}, \tilde{h})$ of $(N, h),{ }^{1}$ the square of distance function $d_{\widetilde{N}}^{2}(\cdot, p)$ is convex for any $p \in \tilde{N}$. If $u \in H^{1}(\Omega, N)$ is a weakly harmonic map, then $u \in \operatorname{Lip}_{\text {loc }}(\Omega, N)$, and for some $0<\alpha<1, u \in C_{\mathrm{loc}}^{1, \alpha}\left(\Omega^{+} \cup \Gamma, N\right) \cap C_{\mathrm{loc}}^{1, \alpha}\left(\Omega^{-} \cup \Gamma, N\right)$.

The idea for the proof of Theorem 1.1 is motivated in [Evans 1991] and [Bethuel 1993]. However, there are several new technical difficulties:

(i) Establishing an almost energy monotonicity inequality for stationary harmonic maps in $(\Omega, g)$. This is achieved by observing that an exact monotonicity inequality holds at any $x \in \Gamma$, see Section 2 below.

\footnotetext{
${ }^{1}$ Here the covering map $\Pi: \widetilde{N} \rightarrow N$ is a Riemannian submersion.
} 
(ii) Establishing a Hodge decomposition in $L^{p}\left(B, \mathbb{R}^{n}\right)$, for any $1<p<+\infty$, on a ball $B=B_{r}(0)$, equipped with certain piecewise continuous metrics $g$. More precisely, we need to show that the solution of

$$
\begin{cases}\frac{\partial}{\partial x_{i}}\left(a_{i j} \frac{\partial v}{\partial x_{j}}\right)=\operatorname{div} f & \text { in } B, \\ v=0 & \text { on } \partial B\end{cases}
$$

enjoys a $W^{1, p}$-estimate: for any $1<p<+\infty$,

$$
\|\nabla v\|_{L^{p}(B)} \leq C\|f\|_{L^{p}(B)}
$$

provided that $\left(a_{i j}\right) \in C\left(\overline{B^{ \pm}}\right) \cap C\left(B^{\delta}\right)$ for some $\delta>0$, is uniformly elliptic, but is discontinuous on $\partial B^{+} \backslash B^{\delta}$, where $B^{\delta}=\{x \in B$ : $\operatorname{dist}(x, \partial B) \leq \delta\}$. This follows from a recent theorem in [Byun and Wang 2010; Dong and Kim 2010]; see also [Dong and Kim 2011a; 2011b] and Section 3 below.

(iii) Employing the moving frame method to establish the decay estimate in suitable Morrey spaces under a smallness condition, analogous to [Ishizuka and Wang 2008]. To obtain Lipschitz and piecewise $C^{1, \alpha}$-regularity, we compare the harmonic map system with an elliptic system with piecewise constant coefficients and perform a hole-filling argument, similar to [Giaquinta and Hildebrandt 1982].

The paper is organized as follows. In Section 2, we derive an almost energy monotonicity inequality. In Section 3 , we show the global $W^{1, p}(1<p<\infty)$ estimate for elliptic systems with certain piecewise continuous coefficients, and a Hodge decomposition theorem. In Section 4, we adapt the moving frame method of [Hélein 2002] and [Bethuel 1993] to establish an $\epsilon$-Hölder continuity. In Section 5, we establish both Lipschitz and piecewise $C^{1, \alpha}$ regularity for Hölder continuous harmonic maps. In Section 6, we consider harmonic maps into manifolds supporting convex distance square functions and prove Theorem 1.2.

\section{Energy monotonicity inequality}

This section is devoted to the derivation of energy monotonicity inequalities for stationary harmonic maps from $(\Omega, g)$ to $(N, h)$.

Theorem 2.1. Under the same assumptions as in Theorem 1.1, there exist $C>0$ and $r_{0}>0$, depending only on $\Omega, \Gamma$, and $g$, such that if $u \in W^{1,2}(\Omega, N)$ is a stationary harmonic map on $(\Omega, g)$, then for any $x_{0} \in \Omega$, there holds

$$
s^{2-n} \int_{B_{s}\left(x_{0}\right)}|\nabla u|_{g}^{2} d v_{g} \leq e^{C r} r^{2-n} \int_{B_{r}\left(x_{0}\right)}|\nabla u|_{g}^{2} d v_{g}
$$

for all $0<s \leq r \leq \min \left\{r_{0}, \operatorname{dist}\left(x_{0}, \partial \Omega\right)\right\}$. 
Since $g \in C^{0,1}\left(\Omega^{ \pm}\right)$, there are $C>0$ and $r_{0}>0$ such that (2-1) holds for any $x_{0} \in \Omega^{ \pm}$and $0<s \leq r \leq \min \left\{r_{0}, \operatorname{dist}\left(x_{0}, \partial \Omega^{ \pm}\right)\right\}$; see [Hélein 2002]. In particular, (2-1) holds for any $x_{0} \in \Omega \backslash \Gamma^{r_{0}}$ and $0<s \leq r \leq \min \left\{r_{0}\right.$, $\left.\operatorname{dist}\left(x_{0}, \partial \Omega\right)\right\}$, where $\Gamma^{r_{0}}=\left\{x \in \Omega\right.$ : $\left.\operatorname{dist}(x, \Gamma) \leq r_{0}\right\}$ is the $r_{0}$-neighborhood of $\Gamma$. To show (2-1) for $x_{0} \in \Gamma^{r_{0}}$, it suffices to consider the case $x_{0} \in \Gamma$.

It follows from the assumption on $\Gamma$ and $g$ that there exists $r_{0}>0$ such that for any $x_{0} \in \Gamma$ there exists a $C^{1,1}$-diffeomorphism $\Phi_{0}: B_{1} \rightarrow B_{r_{1}}\left(x_{0}\right)$, where $r_{1}=\min \left\{r_{0}, \operatorname{dist}\left(x_{0}, \partial \Omega\right)\right\}$, such that

$$
\left\{\begin{array}{l}
\Phi_{0}\left(B_{1}^{ \pm}\right)=\Omega^{ \pm} \cap B_{r_{1}}\left(x_{0}\right), \\
\Phi_{0}\left(\Gamma_{1}\right)=\Gamma \cap B_{r_{1}}\left(x_{0}\right), \text { where } \Gamma_{1}=\left\{x \in B_{1}: x_{n}=0\right\} .
\end{array}\right.
$$

Define $\tilde{u}(x)=u\left(\Phi_{0}(x)\right)$ and $\tilde{g}(x)=\Phi_{0}^{*}(g)(x)$ for $x \in B_{1}$. Then it is readily seen that $\tilde{g}$ is piecewise $C^{0,1}$, with $\Gamma$ as its discontinuity set, and satisfies (1-4) on $\Gamma_{1}$. (In fact, since

$$
\Phi_{0}^{*}(g)_{i j}(x)=g_{k l}\left(\Phi_{0}(x)\right) \frac{\partial \Phi_{0}^{k}}{\partial x_{i}}(x) \frac{\partial \Phi_{0}^{l}}{\partial x_{j}}(x),
$$

condition (1-4) implies that

$$
\lim _{\substack{y \in \Omega^{+} \\ y \rightarrow x}} \Phi_{0}^{*} g(y)=k\left(\Phi_{0}(x)\right) \lim _{\substack{y \in \Omega^{-} \\ y \rightarrow x}} \Phi_{0}^{*} g(y)
$$

for any $x \in \Gamma_{1}$.) It is also easy to see that, if $u:\left(B_{r_{1}}\left(x_{0}\right), g\right) \rightarrow(N, h)$ is a stationary harmonic map, so $\tilde{u}:\left(B_{1}, \tilde{g}\right) \rightarrow(N, h)$.

Thus we may assume that $\Omega=B_{1}$, that $g$ is a piecewise $C^{0,1}$-metric which satisfies (1-4) on the set of discontinuity $\Gamma_{1}$, and that $u:\left(B_{1}, g\right) \rightarrow(N, h)$ is a stationary harmonic map. It suffices to establish (2-1) in $B_{1 / 2}$. We first derive a stationarity identity for $u$.

Proposition 2.2. Let $u \in W^{1,2}\left(B_{1}, N\right)$ be a stationary harmonic map on $\left(B_{1}, g\right)$. Then

$$
\begin{aligned}
& \int_{B_{1}}\left(2 g^{i j}\left\langle\frac{\partial u}{\partial x_{k}}, \frac{\partial u}{\partial x_{j}}\right) Y_{i}^{k}-|\nabla u|_{g}^{2} \operatorname{div} Y\right) \sqrt{g} d x \\
&=\int_{B_{1}} \frac{\partial}{\partial x_{k}}\left(\sqrt{g} g^{i j}\right) Y^{k}\left\langle\frac{\partial u}{\partial x_{i}}, \frac{\partial u}{\partial x_{j}}\right\rangle d x
\end{aligned}
$$

for all $Y=\left(Y^{1}, \ldots, Y^{n-1}, Y^{n}\right) \in C_{0}^{1}\left(B_{1}, \mathbb{R}^{n}\right)$ satisfying

$$
Y^{n}(x) \begin{cases}\geq 0 & \text { for } x^{n}>0 \\ =0 & \text { for } x^{n}=0 \\ \leq 0 & \text { for } x^{n}<0\end{cases}
$$

where $Y_{i}^{k}=\partial Y^{k} / \partial x_{i}$ and $\operatorname{div} Y=\sum_{i=1}^{n} \partial Y^{i} / \partial x_{i}$. 
Proof. Let $Y \in C_{0}^{1}\left(B_{1}, \mathbb{R}^{n}\right)$ satisfy (2-3). Then there exists $\delta>0$ such that $F_{t}(x)=$ $x+t Y(x), t \in[-\delta, \delta]$, is a family of diffeomorphisms from $B_{1}$ to $B_{1}$ satisfying the condition (1-3). Hence

$$
\begin{aligned}
0 & =\left.\frac{d}{d t}\right|_{t=0} \int_{B_{1}}\left|\nabla u\left(F_{t}(x)\right)\right|_{g}^{2} d v_{g} \\
& =\left.\frac{d}{d t}\right|_{t=0}\left(\int_{B_{1}^{+}}\left|\nabla u\left(F_{t}(x)\right)\right|_{g}^{2} d v_{g}+\int_{B_{1}^{-}}\left|\nabla u\left(F_{t}(x)\right)\right|_{g}^{2} d v_{g}\right) .
\end{aligned}
$$

Set $G_{t}=F_{t}^{-1}$, for $t \in[-\delta, \delta]$. Direct calculations yield

$$
\begin{aligned}
& \left.\frac{d}{d t}\right|_{t=0} \int_{B_{1}^{ \pm}} \mid \nabla\left(\left.u\left(F_{t}(x)\right)\right|_{g} ^{2} d v_{g}\right. \\
& =\left.\frac{d}{d t}\right|_{t=0} \int_{B_{1}^{ \pm}} \sqrt{g(x+t Y(x))} g^{i j}(x+t Y(x))\left\langle\frac{\partial u}{\partial x_{k}}, \frac{\partial u}{\partial x_{l}}\right\rangle \\
& \quad \times(x+t Y(x))\left(\delta_{k i}+t Y_{i}^{k}\right)\left(\delta_{l j}+t Y_{j}^{l}\right) d x \\
& =\int_{B_{1}^{ \pm}} \sqrt{g(x)} g^{i j}(x)\left\langle\frac{\partial u}{\partial x_{k}}, \frac{\partial u}{\partial x_{l}}\right\rangle\left(\delta_{k i} Y_{j}^{l}+\delta_{l j} Y_{i}^{k}\right) d x \\
& \quad+\left.\int_{B_{1}^{ \pm}} \frac{d}{d t}\right|_{t=0}\left(g^{i j}\left(G_{t}(x)\right) \sqrt{g\left(G_{t}(x)\right)} J G_{t}(x)\right)\left\langle\frac{\partial u}{\partial x_{i}}, \frac{\partial u}{\partial x_{j}}\right\rangle d x \\
& =\int_{B_{1}^{ \pm}}\left(2 g^{i j}\left\langle\frac{\partial u}{\partial x_{i}}, \frac{\partial u}{\partial x_{l}}\right\rangle Y_{j}^{l}-g^{i j}\left\langle\frac{\partial u}{\partial x_{i}}, \frac{\partial u}{\partial x_{j}}\right\rangle \operatorname{div} Y\right) \sqrt{g} d x \\
& \quad-\int_{B_{1}^{ \pm}} \frac{\partial}{\partial x_{k}}\left(\sqrt{g} g^{i j}\right) Y^{k}\left\langle\frac{\partial u}{\partial x_{i}}, \frac{\partial u}{\partial x_{j}}\right\rangle d x,
\end{aligned}
$$

where we have used the equalities

$$
\left\{\begin{array}{l}
\left.\frac{d}{d t}\right|_{t=0} J G_{t}(x)=-\operatorname{div} Y, \\
\left.\frac{d}{d t}\right|_{t=0} G_{t}(x)=-Y(x), \\
\left.\frac{d}{d t}\right|_{t=0}\left(g^{i j}\left(G_{t}(x)\right) \sqrt{g\left(G_{t}(x)\right)}\right)=-\frac{\partial}{\partial x_{k}}\left(\sqrt{g} g^{i j}\right) Y^{k} .
\end{array}\right.
$$

This completes the proof.

Proposition 2.3. Let $u \in W^{1,2}\left(B_{1}, N\right)$ be a stationary harmonic map on $\left(B_{1}, g\right)$. There exists $C>0$ such that:

(i) For any $x_{0}=\left(x_{0}^{\prime}, x_{0}^{n}\right) \in B_{1 / 2} \backslash \Gamma_{1}$, there exists $0<R_{0} \leq \min \left\{\frac{1}{4},\left|x_{0}^{n}\right|\right\}$ such that

$$
r^{2-n} \int_{B_{r}\left(x_{0}\right)}|\nabla u|_{g}^{2} d v_{g} \leq e^{C R} R^{2-n} \int_{B_{R}\left(x_{0}\right)}|\nabla u|_{g}^{2} d v_{g} \quad \text { if } 0<r \leq R<R_{0} .
$$


(ii) For any $x_{0} \in B_{1 / 2}$,

$$
r^{2-n} \int_{B_{r}\left(x_{0}\right)}|\nabla u|_{g}^{2} d v_{g} \leq e^{C R} R^{2-n} \int_{B_{R}\left(x_{0}\right)}|\nabla u|_{g}^{2} d v_{g} \quad \text { if } 0<r \leq R \leq \frac{1}{4} .
$$

Proof. (i) By choosing $Y \in C_{c}^{\infty}\left(B_{1}^{+}, \mathbb{R}^{n}\right)$ or $Y \in C_{c}^{\infty}\left(B_{1}^{-}, \mathbb{R}^{n}\right)$, we conclude that $u$ is a stationary harmonic map on $\left(B_{1}^{+}, g\right)$ and $\left(B_{1}^{-}, g\right)$. Hence the monotonicity inequality (2-4) holds; see [Hélein 2002].

(ii) Step 1. We first consider the case where $x_{0} \in \Gamma_{1}$. Without loss of generality, we can assume that $x_{0}=\left(0^{\prime}, 0\right)$. For $\epsilon>0$ and $0<r \leq \frac{1}{2}$, let $Y_{\epsilon}(x)=x \eta_{\epsilon}(x)$, where $\eta_{\epsilon}(x)=\eta_{\epsilon}(|x|) \in C_{0}^{\infty}\left(B_{1}\right)$ satisfies

$$
0 \leq \eta_{\epsilon} \leq 1, \quad \eta_{\epsilon}^{\prime} \leq 0, \quad\left|\eta_{\epsilon}^{\prime}\right| \leq \frac{2}{\epsilon}, \quad \eta_{\epsilon}(s)= \begin{cases}1 & \text { for } 0 \leq s \leq r-\epsilon \\ 0 & \text { for } s \geq r .\end{cases}
$$

Then

$$
\left(Y_{\epsilon}\right)_{i}^{j}=\delta_{i j} \eta_{\epsilon}(|x|)+\eta_{\epsilon}^{\prime}(|x|) \frac{x^{i} x^{j}}{|x|} .
$$

Substituting $Y_{\epsilon}$ into the right side of (2-2), and using

$$
\left|\frac{\partial}{\partial x_{k}}\left(\sqrt{g} g^{i j}\right)\right| \leq C \quad \text { for a.e. } x \in B_{1} \backslash \Gamma_{1},
$$

we have

$$
\begin{aligned}
\left|\int_{B_{1}} \frac{\partial}{\partial x_{k}}\left(\sqrt{g} g^{i j}\right) Y_{\epsilon}^{k}\left\langle\frac{\partial u}{\partial x_{i}}, \frac{\partial u}{\partial x_{j}}\right\rangle d x\right| & \leq C r \int_{B_{r}}|\nabla u|^{2} d x \\
& \leq C r \int_{B_{r}}|\nabla u|_{g}^{2} d v_{g} .
\end{aligned}
$$

Substituting (2-7) into the left side of (2-2), we obtain

$$
\begin{aligned}
\int_{B_{1}}\left(2 g^{i j}\left\langle\frac{\partial u}{\partial x_{j}}, \frac{\partial u}{\partial x_{k}}\right\rangle\left(Y_{\epsilon}\right)_{i}^{k}-|\nabla u|_{g}^{2} \operatorname{div} Y_{\epsilon}\right) \sqrt{g} d x & \\
=(2-n) \int_{B_{1}}|\nabla u|_{g}^{2} \eta_{\epsilon}(|x|) & \sqrt{g} d x-\int_{B_{1}}|\nabla u|_{g}^{2}|x| \eta_{\epsilon}^{\prime}(|x|) \sqrt{g} d x \\
& +\int_{B_{1}} 2 g^{i j}\left\langle\frac{\partial u}{\partial x_{i}}, \frac{\partial u}{\partial x_{k}}\right\rangle \frac{x^{k} x^{j}}{|x|} \eta_{\epsilon}^{\prime}(|x|) \sqrt{g} d x .
\end{aligned}
$$

Define $\bar{g}$ by

$$
\bar{g}\left(x^{\prime}, x^{n}\right)= \begin{cases}\lim _{y \rightarrow 0, y^{n} \geq 0} g(y) & \text { if } x^{n} \geq 0, \\ \lim _{y \rightarrow 0, y^{n}<0} g(y) & \text { if } x^{n}<0 .\end{cases}
$$

Then we have

$$
|g(x)-\bar{g}(x)| \leq C|x| \text { for all } x \in B_{1} \text {. }
$$


Further, by (1-4) we can assume

$$
\bar{g}(x)= \begin{cases}g_{0} & \text { if } x^{n} \geq 0 \\ k g_{0} & \text { if } x^{n}<0 \quad(k \neq 1) .\end{cases}
$$

Hence we can write

$$
\int_{B_{1}} 2 g^{i j}\left\langle\frac{\partial u}{\partial x_{i}}, \frac{\partial u}{\partial x_{k}}\right\rangle \frac{x^{k} x^{j}}{|x|} \eta_{\epsilon}^{\prime}(|x|) \sqrt{g} d x=I_{\epsilon}+I I_{\epsilon} .
$$

where

$$
\begin{aligned}
I_{\epsilon} & =2 \int_{B_{1}} \bar{g}^{i j}\left\langle\frac{\partial u}{\partial x_{i}}, \frac{\partial u}{\partial x_{k}}\right\rangle \frac{x^{k} x^{j}}{|x|} \eta_{\epsilon}^{\prime}(|x|) \sqrt{g} d x, \\
I I_{\epsilon} & =2 \int_{B_{1}}\left(g^{i j}-\bar{g}^{i j}\right)\left\langle\frac{\partial u}{\partial x_{i}}, \frac{\partial u}{\partial x_{k}}\right\rangle \frac{x^{k} x^{j}}{|x|} \eta_{\epsilon}^{\prime}(|x|) \sqrt{g} d x .
\end{aligned}
$$

Since

$$
\bar{g}^{i j}\left\langle\frac{\partial u}{\partial x_{i}}, \frac{\partial u}{\partial x_{k}}\right\rangle \frac{x^{k} x^{j}}{|x|}= \begin{cases}|x||\partial u / \partial r|^{2} & \text { if } x^{n} \geq 0 \\ (1 / k)|x||\partial u / \partial r|^{2} & \text { if } x^{n}<0\end{cases}
$$

is nonnegative in $B_{1}$ and $\eta_{\epsilon}^{\prime}(|x|) \leq 0$, we have $I_{\epsilon} \leq 0$. For $I I_{\epsilon}$, by (2-10) we have

$$
\left|I I_{\epsilon}\right| \leq C r^{2} \int_{B_{r}}|\nabla u|_{g}^{2}\left|\eta_{\epsilon}^{\prime}\right|(|x|) d v_{g} .
$$

Putting these estimates first into (2-11) and then into (2-9), and finally combining (2-9) and (2-8) with (2-2), we obtain, after taking $\epsilon$ to zero,

$$
\begin{aligned}
(2-n) \int_{B_{r}}|\nabla u|_{g}^{2} d v_{g} & +r \int_{\partial B_{r}}|\nabla u|_{g}^{2} \sqrt{g} d H^{n-1} \\
\geq & -C\left(r \int_{B_{r}}|\nabla u|_{g}^{2} d v_{g}+r^{2} \int_{\partial B_{r}}|\nabla u|_{g}^{2} \sqrt{g} d H^{n-1}\right) .
\end{aligned}
$$

It is not hard to see that (2-13) implies

$$
\frac{d}{d r}\left(e^{C r} r^{2-n} \int_{B_{r}}|\nabla u|_{g}^{2} d v_{g}\right) \geq 0
$$

so that (2-5) holds when $x_{0} \in B_{1 / 2}$.

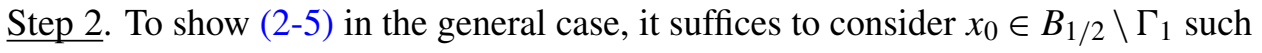
that

$$
\left|B_{R}\left(x_{0}\right) \cap B_{1}^{+}\right|>0 \quad \text { and } \quad\left|B_{R}\left(x_{0}\right) \cap B_{1}^{-}\right|>0 .
$$

For simplicity, assume $x_{0} \in B_{1}^{-}$. We consider two cases:

Suppose $d\left(x_{0}, \Gamma_{1}\right)=\left|x_{0}^{n}\right| \geq \frac{1}{4} R$. Then: 
- If $R \geq r \geq \frac{1}{4} R$, it is easy to see that

$$
r^{2-n} \int_{B_{r}\left(x_{0}\right)}|\nabla u|_{g}^{2} d v_{g} \leq 4^{n-2} R^{2-n} \int_{B_{R}\left(x_{0}\right)}|\nabla u|_{g}^{2} d v_{g}
$$

- If $0<r<\frac{1}{4} R\left(\leq d\left(x_{0}, \Gamma_{1}\right)\right)$, we have $B_{R / 4}\left(x_{0}\right) \subset B_{1}^{-}$, so (2-4) implies

$$
\begin{aligned}
r^{2-n} \int_{B_{r}\left(x_{0}\right)}|\nabla u|_{g}^{2} d v_{g} & \leq e^{C R}\left(\frac{R}{4}\right)^{2-n} \int_{B_{R / 4}\left(x_{0}\right)}|\nabla u|_{g}^{2} d v_{g} \\
& \leq e^{C R} R^{2-n} \int_{B_{R}\left(x_{0}\right)}|\nabla u|_{g}^{2} d v_{g} .
\end{aligned}
$$

Suppose instead that $d\left(x_{0}, \Gamma_{1}\right)=\left|x_{0}^{n}\right|<\frac{1}{4} R$. Then:

- If $R \geq r \geq \frac{1}{4} R$, then

$$
r^{2-n} \int_{B_{r}\left(x_{0}\right)}|\nabla u|_{g}^{2} d v_{g} \leq 4^{n-2} R^{2-n} \int_{B_{R}\left(x_{0}\right)}|\nabla u|_{g}^{2} d v_{g}
$$

- If $0<r \leq d\left(x_{0}, \Gamma_{1}\right)=\left|x_{0}^{n}\right|<\frac{1}{4} R$, then by setting $\bar{x}_{0}=\left(x_{0}^{1}, \ldots, x_{0}^{n-1}, 0\right)$ we have

$$
B_{r}\left(x_{0}\right) \subset B_{\left|x_{0}^{n}\right|}\left(x_{0}\right) \subset B_{2\left|x_{0}^{n}\right|}\left(\bar{x}_{0}\right) \subset B_{R / 2}\left(\bar{x}_{0}\right) \subset B_{R}\left(x_{0}\right),
$$

so that (2-5) yields

$$
\begin{aligned}
r^{2-n} \int_{B_{r}\left(x_{0}\right)}|\nabla u|_{g}^{2} d v_{g} & \leq\left|x_{0}^{n}\right|^{2-n} \int_{B_{\left|x_{0}^{n}\right|}\left(x_{0}\right)}|\nabla u|_{g}^{2} d v_{g} \\
& \leq 2^{n-2}\left(2\left|x_{0}^{n}\right|\right)^{2-n} \int_{B_{2\left|x_{0}^{n}\right|}\left(\bar{x}_{0}\right)}|\nabla u|_{g}^{2} d v_{g} \\
& \leq 2^{n-2} e^{C R}\left(\frac{R}{2}\right)^{2-n} \int_{B_{R / 2}\left(\bar{x}_{0}\right)}|\nabla u|_{g}^{2} d v_{g} \\
& \leq e^{C R} R^{2-n} \int_{B_{R}\left(x_{0}\right)}|\nabla u|_{g}^{2} d v_{g} .
\end{aligned}
$$

- If $d\left(x_{0}, \Gamma_{1}\right)\left(=\left|x_{0}^{n}\right|\right) \leq r<\frac{1}{4} R$, then we have

$$
B_{r}\left(x_{0}\right) \subset B_{2 r}\left(\bar{x}_{0}\right) \subset B_{R / 2}\left(\bar{x}_{0}\right) \subset B_{R}\left(x_{0}\right),
$$


so that (2-5) yields

$$
\begin{aligned}
r^{2-n} \int_{B_{r}\left(x_{0}\right)}|\nabla u|_{g}^{2} d v_{g} & \leq 2^{n-2}(2 r)^{2-n} \int_{B_{2 r}\left(\bar{x}_{0}\right)}|\nabla u|_{g}^{2} d v_{g} \\
& \leq 2^{n-2} e^{C R}\left(\frac{R}{2}\right)^{2-n} \int_{B_{R / 2}\left(\bar{x}_{0}\right)}|\nabla u|_{g}^{2} d v_{g} \\
& \leq e^{C R} R^{2-n} \int_{B_{R}\left(x_{0}\right)}|\nabla u|_{g}^{2} d v_{g} .
\end{aligned}
$$

Therefore (2-5) is proved in all cases.

\section{3. $W^{1, p}$-estimate for elliptic equations with piecewise continuous coefficients}

In this section, we will provide the global $W^{1, p}$-estimate for elliptic equations with piecewise continuous coefficients. The proof is a slight modification of that of [Dong and Kim 2010] (see also [Dong and Kim 2011a; 2011b]) or [Byun and Wang 2010]. As a corollary, we will establish the Hodge decomposition theorem (Theorem 3.2) for piecewise continuous metrics $g$, a crucial ingredient to prove Theorem 1.1.

For a ball $B=B_{r}(0) \subset \mathbb{R}^{n}$, set $B^{\epsilon}=\{x \in B: \operatorname{dist}(x, \partial B) \leq \epsilon\}$ for $\epsilon>0$. Let $\left(a_{i j}(x)\right)_{1 \leq i, j \leq n}$ be bounded measurable, uniformly elliptic on $B$; i.e., there exist $0<\lambda \leq \Lambda<+\infty$ such that

$$
\lambda|\xi|^{2} \leq a_{i j}(x) \xi_{i}^{\alpha} \xi_{\beta}^{j} \leq \Lambda|\xi|^{2} \text { a.e. } x \in B \text { for all } \xi \in \mathbb{R}^{n} .
$$

Theorem 3.1. Assume $\left(a_{i j}\right)$ satisfies (3-1), and there exists $\epsilon>0$ such that $\left(a_{i j}\right) \in$ $C\left(\overline{B^{ \pm}}\right) \cap C\left(B^{\epsilon}\right)$ and is discontinuous on $\partial B^{+} \backslash B^{\epsilon}$. For $p \in(1,+\infty)$, let $f \in$ $L^{p}\left(B, \mathbb{R}^{n}\right)$. Then there exists a unique weak solution $v \in W_{0}^{1, p}\left(B, \mathbb{R}^{n}\right)$ to

$$
\begin{cases}\sum_{i, j} \frac{\partial}{\partial x_{i}}\left(a_{i j} \frac{\partial v}{\partial x_{j}}\right)=\sum_{i} \frac{\partial f_{i}}{\partial x_{i}} & \text { in } B, \\ u=0 & \text { on } \partial B\end{cases}
$$

that satisfies

$$
\|\nabla v\|_{L^{p}(B)} \leq C\|f\|_{L^{p}(B)}
$$

for some $C>0$ depending only on $p$ and $\left(a_{i j}\right)$.

Proof. By (3-1), we see that for any $\delta>0$, there exists $R=R(\delta)>0$ such that the coefficient function $\left(a_{i j}\right)$ satisfies the $(\delta, R)$-vanishing of codimension-one conditions (2.5) and (2.6) of [Byun and Wang 2010, p. 2562]; see also [Dong and Kim 2010; 2011a; 2011b]. In fact, we have

$$
\lim _{r \downarrow 0} \max _{x_{0}=\left(x_{0}^{\prime}, x_{0}^{n}\right) \in \bar{B}}\left\|a_{i j}\left(x^{\prime}, x^{n}\right)-a_{i j}\left(x_{0}^{\prime}, x^{n}\right)\right\|_{L^{\infty}\left(B_{r}\left(\left(x_{0}^{\prime}, x_{0}^{n}\right)\right)\right)}=0 .
$$


Therefore Theorem 3.1 follows directly from [Byun and Wang 2010, Theorem 2.2, p. 2653].

As an immediate consequence of Theorem 3.1, we have the following Hodge decomposition on $B$ equipped with certain piecewise continuous metrics $g$.

Theorem 3.2. Let $\bar{g}$ be a piecewise continuous metric on $B$ such that $\bar{g}$ is continuous on $B^{ \pm}$and on $B^{\delta}$ for some $\delta>0$, and is discontinuous on $\partial B^{+} \backslash B^{\delta}$. Then for any $p \in(1,+\infty)$ and $F=\left(F_{1}, \ldots, F_{n}\right) \in L^{p}\left(B, \mathbb{R}^{n}\right)$, there exist $G \in W_{0}^{1, p}(B)$ and $H \in L^{p}\left(B, \mathbb{R}^{n}\right)$ such that

$$
F=\nabla G+H, \operatorname{div}_{\bar{g}} H\left(:=\frac{1}{\sqrt{\bar{g}}} \frac{\partial}{\partial x_{i}}\left(\sqrt{\bar{g}} \bar{g}^{i j} H_{j}\right)\right)=0 \quad \text { in } B .
$$

Further, there exists $C=C(p, n, \bar{g})>0$ such that

$$
\|\nabla G\|_{L^{p}(B)}+\|H\|_{L^{p}(B)} \leq C\|F\|_{L^{p}(B)} .
$$

Proof. For $1 \leq i, j \leq n$, set $a_{i j}=\sqrt{\bar{g}} \bar{g}^{i j}$ on $B$. Then $\left(a_{i j}\right)$ satisfies the conditions of Theorem 3.1, so that there exists a unique solution $G \in W_{0}^{1, p}(B)$ to

$$
\begin{cases}\frac{\partial}{\partial x_{i}}\left(\sqrt{\bar{g}} \bar{g}^{i j} \frac{\partial G}{\partial x_{j}}\right)=\frac{\partial}{\partial x_{i}}\left(\sqrt{\bar{g}} \bar{g}^{i j} F_{j}\right) & \text { in } B, \\ G=0 & \text { on } \partial B,\end{cases}
$$

and

$$
\|\nabla G\|_{L^{p}(B)} \leq C\left\|\sqrt{\bar{g}} \bar{g}^{i j} F_{j}\right\|_{L^{p}(B)} \leq C\|F\|_{L^{p}(B)} .
$$

Set $H=F-\nabla G$. Then we have

$$
\operatorname{div}_{\bar{g}} H=\frac{1}{\sqrt{\bar{g}}} \frac{\partial}{\partial x_{i}}\left(\sqrt{\bar{g}} \bar{g}^{i j}\left(F_{j}-\frac{\partial G}{\partial x_{j}}\right)\right)=0 \text { on } B,
$$

and

$$
\|H\|_{L^{p}\left(B_{1 / 2}\right)} \leq\|F\|_{L^{p}\left(B_{1 / 2}\right)}+\|\nabla G\|_{L^{p}(B)} \leq C\|F\|_{L^{p}(B)} .
$$

This completes the proof.

\section{Hölder continuity}

In this section, we will prove that any stationary harmonic map on $\left(B_{1}, g\right)$, with $g \in C^{0,1}\left(B_{1}^{ \pm} \cup \Gamma_{1}\right)$, is Hölder continuous provided that $\int_{B_{1}}|\nabla u|_{g}^{2} d v_{g}$ is sufficiently small. The idea is based on suitable modifications of the original argument in [Bethuel 1993] (see also [Ishizuka and Wang 2008]), thanks to both the energy monotonicity inequality and the Hodge decomposition theorem established in the previous two sections. More precisely: 
Theorem 4.1. There exist $\epsilon_{0}>0$ and $\alpha_{0} \in(0,1)$, depending only on $n, g$, such that if the metric $g \in C^{0,1}\left(B_{1}^{ \pm} \cup \Gamma_{1}\right)$ satisfies the condition (1-4) on $\Gamma_{1}$, and $u \in$ $W^{1,2}\left(B_{1}, N\right)$ is a stationary harmonic map satisfying

$$
r_{0}^{2-n} \int_{B_{r_{0}}\left(x_{0}\right)}|\nabla u|_{g}^{2} d v_{g} \leq \epsilon_{0}^{2}
$$

for some $x_{0} \in B_{1 / 2}$ and $0<r_{0} \leq \frac{1}{4}$, then $u \in C^{\alpha_{0}}\left(B_{r_{0} / 2}\left(x_{0}\right), N\right)$, and

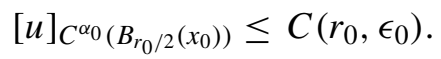

Proof of Theorem 4.1. The proof is based on suitable modifications of [Bethuel 1993; Ishizuka and Wang 2008]. First, observe that if $x_{0}=\left(x_{0}^{\prime}, x_{0}^{n}\right) \in B^{ \pm}$, it follows from the monotonicity inequality (2-5) that we may assume (4-1) holds for some $0<r_{0}<\left|x_{0}^{n}\right|$. Then the $\epsilon_{0}$-regularity theorem in [Bethuel 1993] (see [Ishizuka and Wang 2008] for domains with $C^{0,1}$ metrics) implies that for some $0<\alpha_{0}<1, u \in C^{\alpha_{0}}\left(B_{r_{0} / 2}\left(x_{0}\right)\right)$ and (4-2) holds. Hence it suffices to consider the case $x_{0}=\left(x_{0}^{\prime}, 0\right) \in \Gamma_{1 / 2}$. By translation and scaling, we may assume $x_{0}=(0,0)$ and proceed as follows.

Step 1. As in [Bethuel 1993; Hélein 2002; Ishizuka and Wang 2008], we assume that there exists an orthonormal frame on $\left.u^{*} T N\right|_{B_{1}}$. For $0<\theta<\frac{1}{2}$, to be determined later, let $\left\{e_{\alpha}\right\}_{\alpha=1}^{l} \subset W^{1,2}\left(B_{2 \theta}, \mathbb{R}^{k}\right)$ be a Coulomb gauge orthonormal frame of $\left.u^{*} T N\right|_{B_{2 \theta}}$; that is,

$$
\begin{gathered}
\operatorname{div}_{g}\left(\left\langle\nabla e_{\alpha}, e_{\beta}\right\rangle\right)=0 \quad \text { in } B_{2 \theta} \quad(1 \leq \alpha, \beta \leq l), \\
\sum_{\alpha=1}^{l} \int_{B_{2 \theta}}\left|\nabla e_{\alpha}\right|_{g}^{2} d v_{g} \leq C \int_{B_{2 \theta}}|\nabla u|_{g}^{2} d v_{g} .
\end{gathered}
$$

For $1 \leq \alpha \leq l$, consider $\left\langle\nabla\left(\left(u-u_{2 \theta}\right) \eta\right), e_{\alpha}\right\rangle$, where $u_{2 \theta}=f_{B_{2 \theta}} u$ is the average of $u$ on $B_{2 \theta}$, and $\eta \in C_{0}^{\infty}\left(B_{1}\right)$ satisfies

$$
0 \leq \eta \leq 1 ; \quad \eta=1 \text { in } B_{\theta} ; \quad \eta=0 \text { outside } B_{7 \theta / 4} ; \quad|\nabla \eta| \leq \frac{2}{\theta} .
$$

Define the metric $\tilde{g}$ on $B_{2 \theta}$ by

$$
\tilde{g}(x)=\eta(x) g(x)+(1-\eta(x)) g_{0}(x), \quad x \in B_{2 \theta} .
$$

Then it is easy to see that

$$
\tilde{g} \equiv g \text { on } B_{\theta} ; \quad \tilde{g} \equiv g_{0} \text { outside } B_{7 \theta / 4} ; \quad \tilde{g} \in C\left(\overline{B_{2 \theta}^{ \pm}}\right) \cap C\left(B_{2 \theta} \backslash B_{7 \theta / 4}\right) .
$$

In particular, $\tilde{g}$ satisfies the conditions of Theorem 3.2. Hence, by Theorem 3.2, for 
$1<p<n /(n-1)$, there exist $\phi_{\alpha} \in W_{0}^{1, p}\left(B_{2 \theta}\right)$ and $\psi_{\alpha} \in L^{p}\left(B_{2 \theta}\right)$ such that

$$
\left\langle\nabla\left(\left(u-u_{2 \theta}\right) \eta\right), e_{\alpha}\right\rangle=\nabla \phi_{\alpha}+\psi_{\alpha}, \quad \operatorname{div}_{\tilde{g}}\left(\psi_{\alpha}\right)=0 \quad \text { in } B_{2 \theta},
$$$$
\left\|\nabla \phi_{\alpha}\right\|_{L^{p}\left(B_{2 \theta}\right)}+\left\|\psi_{\alpha}\right\|_{L^{p}\left(B_{2 \theta}\right)} \lesssim\left\|\nabla\left(\left(u-u_{2 \theta}\right) \eta\right)\right\|_{L^{p}\left(B_{2 \theta}\right)} \lesssim\|\nabla u\|_{L^{p}\left(B_{2 \theta}\right)} \text {. }
$$

Since $u$ satisfies the harmonic map equation (1-1), we have

$$
\operatorname{div}_{g}\left(\left\langle\nabla u, e_{\alpha}\right\rangle\right)=g^{i j} \nabla_{i} u\left\langle\nabla_{j} e_{\alpha}, e_{\beta}\right\rangle e_{\beta} \quad \text { in } B_{1} .
$$

Thus we obtain

$$
\Delta_{g} \phi_{\alpha}=g^{i j} \nabla_{i} u\left\langle\nabla_{j} e_{\alpha}, e_{\beta}\right\rangle e_{\beta} \quad \text { in } B_{\theta} .
$$

Decompose $\phi_{\alpha}=\phi_{\alpha}^{(1)}+\phi_{\alpha}^{(2)}$, where $\phi_{\alpha}^{(1)}$ solves

$$
\begin{cases}\Delta_{g} \phi_{\alpha}^{(1)}=0 & \text { in } B_{\theta} \\ \phi_{\alpha}^{(1)}=\phi_{\alpha} & \text { on } \partial B_{\theta}\end{cases}
$$

and $\phi_{\alpha}^{(2)}$ solves

$$
\begin{cases}\Delta_{g} \phi_{\alpha}^{(2)}=g^{i j} \nabla_{i} u\left\langle\nabla_{j} e_{\alpha}, e_{\beta}\right\rangle e_{\beta} & \text { in } B_{\theta}, \\ \phi_{\alpha}^{(2)}=0 & \text { on } \partial B_{\theta} .\end{cases}
$$

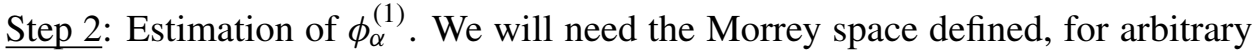
$E \subset \mathbb{R}^{n}$, by

$M^{p, p}(E):=\left\{f: E \rightarrow \mathbb{R} \mid\|f\|_{M^{p, p}(E)}^{p}:=\sup _{B_{r}(x) \subset \mathbb{R}^{n}}\left\{r^{p-n} \int_{B_{r}(x) \cap E}|f|^{p} d x\right\}<+\infty\right\}$.

It is well-known (see [Gilbarg and Trudinger 1983]) that $\phi_{\alpha}^{(1)} \in C^{\alpha_{0}}\left(B_{\theta}\right)$ for some $\alpha_{0} \in(0,1)$, and for any $0<r \leq \theta / 2$,

$$
\left[\phi_{\alpha}^{(1)}\right]_{C^{\alpha_{0}\left(B_{r / 2}\right)}}^{p} \lesssim \theta^{p-n} \int_{B_{\theta}}\left|\nabla \phi_{\alpha}^{(1)}\right|^{p} d x \leq C \theta^{p-n} \int_{B_{2 \theta}}|\nabla u|^{p} d x,
$$

and

$$
(\tau \theta)^{p-n} \int_{B_{\tau \theta}}\left|\nabla \phi_{\alpha}^{(1)}\right|^{p} \leq C \tau^{p \alpha_{0}}\|\nabla u\|_{M^{p, p}\left(B_{1}\right)} \quad \text { for all } 0<\tau<1,
$$

Step 3: Estimation of $\phi_{\alpha}^{(2)}$. Denote by $\mathscr{H}^{1}\left(\mathbb{R}^{n}\right)$ the Hardy space on $\mathbb{R}^{n}$ and $B M O(E)$ the BMO space on $E$ for any open set $E \subset \mathbb{R}^{n}$. By (4.13) of [Ishizuka and Wang 2008, p. 435], for $p^{\prime}=p /(p-1)>n$, there exists $h \in W_{0}^{1, p^{\prime}}\left(B_{\theta}\right)$, with $\|\nabla h\|_{L^{p^{\prime}\left(B_{\theta}\right)}}=1$, such that

$$
\left\|\nabla \phi_{\alpha}^{(2)}\right\|_{L^{p}\left(B_{\theta}\right)} \leq C \int_{B_{\theta}}\left\langle\nabla \phi_{\alpha}^{(2)}, \nabla h\right\rangle_{g} d v_{g}
$$


Using (4-8), (4-4), and the duality between $\mathscr{H}^{1}$ and BMO, we show that

$$
\begin{aligned}
\left\|\nabla \phi_{\alpha}^{(2)}\right\|_{L^{p}\left(B_{\theta}\right)} & \leq C \int_{B_{\theta}} \sqrt{g} g^{i j}\left\langle\nabla_{i} u,\left\langle\nabla_{j} e_{\alpha}, e_{\beta}\right\rangle\right\rangle\left(e_{\beta} h\right) d x \\
& =-C \int_{B_{\theta}} \sqrt{g} g^{i j}\left\langle\nabla_{j} e_{\alpha}, e_{\beta}\right\rangle \nabla_{i}\left(e_{\beta} h\right) u d x \\
& \leq C\left\|\sqrt{g} g^{i j}\left\langle\nabla_{j} e_{\alpha}, e_{\beta}\right\rangle \nabla_{i}\left(e_{\beta} h\right)\right\|_{\mathscr{H}^{1}\left(\mathbb{R}^{n)}\right)}[u]_{B M O\left(B_{\theta}\right)} \\
& \lesssim\left\|\sqrt{g} g^{i j}\left\langle\nabla_{j} e_{\alpha}, e_{\beta}\right\rangle\right\|_{L^{2}\left(B_{\theta}\right)}\left\|\nabla\left(e_{\beta} h\right)\right\|_{L^{2}\left(B_{\theta}\right)}[u]_{B M O\left(B_{\theta}\right)} \\
& \lesssim\|\nabla u\|_{L^{2}\left(B_{2 \theta}\right)}\|\nabla u\|_{M^{p, p}\left(B_{1}\right)} \cdot \theta^{n / p-n / 2} .
\end{aligned}
$$

(Here, to go from the third line to the fourth, we used that $h \in W_{0}^{1, p^{\prime}}\left(B_{\theta}\right)$ and that $\operatorname{div}_{g}\left\langle\nabla e_{\alpha}, e_{\beta}\right\rangle$ vanishes in $B_{\theta}$, so $\sqrt{g} g^{i j}\left\langle\nabla_{j} e_{\alpha}, e_{\beta}\right\rangle \nabla_{i}\left(e_{\beta} h\right) \in \mathscr{H}^{1}\left(\mathbb{R}^{n}\right)$ and $\left\|\sqrt{g} g^{i j}\left\langle\nabla_{j} e_{\alpha}, e_{\beta}\right\rangle \nabla_{i}\left(e_{\beta} h\right)\right\|_{\mathscr{H}^{1}\left(\mathbb{R}^{n}\right)} \leq C\left\|\sqrt{g} g^{i j}\left\langle\nabla_{j} e_{\alpha}, e_{\beta}\right\rangle\right\|_{L^{2}\left(B_{\theta}\right)}\left\|\nabla_{i}\left(e_{\beta} h\right)\right\|_{L^{2}\left(B_{\theta}\right)}$.

This last factor satisfies

$$
\left\|\nabla\left(e_{\beta} h\right)\right\|_{L^{2}\left(B_{\theta}\right)} \leq\left\|\nabla e_{\beta}\right\|_{L^{2}\left(B_{\theta}\right)}\|h\|_{L^{\infty}\left(B_{\theta}\right)}+\|\nabla h\|_{L^{p}\left(B_{\theta}\right)} \theta^{n / p-n / 2} \leq C \theta^{n / p-n / 2},
$$

since the Sobolev embedding implies (because $p^{\prime}>n$ ) that $h \in C^{1-n / p^{\prime}}\left(B_{\theta}\right)$ and

$$
\|h\|_{L^{\infty}\left(B_{\theta}\right)} \leq C \theta^{1-n / p^{\prime}} .
$$

Finally, the estimate $[u]_{B M O\left(B_{\theta}\right)} \leq C\|\nabla u\|_{M^{p, p}\left(B_{1}\right)}$ is a consequence of the Poincaré inequality.)

Putting the estimates of $\phi_{\alpha}^{(1)}$ and $\phi_{\alpha}^{(2)}$ together, we obtain that, for all $0<\tau<1$,

$$
\left((\tau \theta)^{p-n} \int_{B_{\tau \theta}}\left|\nabla \phi_{\alpha}\right|^{p} d x\right)^{1 / p} \leq C\left(\tau^{\alpha_{0}}+\tau^{1-n / p} \epsilon_{0}\right)\|\nabla u\|_{M^{p, p}\left(B_{1}\right)} .
$$

Step 4: Estimation of $\psi_{\alpha}$. Since $\operatorname{div}_{\tilde{g}}\left(\psi_{\alpha}\right)=0$ on $B_{2 \theta}$, we have

$$
\begin{aligned}
\int_{B_{2 \theta}}\left|\psi_{\alpha}\right|_{\tilde{g}}^{2} d v_{\tilde{g}} & =\int_{B_{2 \theta}}\left\langle\psi_{\alpha}+\nabla \phi_{\alpha}, \psi_{\alpha}\right\rangle_{\tilde{g}} d v_{\tilde{g}} \\
& =\int_{B_{2 \theta}}\left\langle\left\langle\nabla\left(\left(u-u_{2 \theta}\right) \eta\right), e_{\alpha}\right\rangle, \psi_{\alpha}\right\rangle_{\tilde{g}} d v_{\tilde{g}} \\
& =-\int_{B_{2 \theta}}\left(u-u_{2 \theta}\right) \eta\left\langle\nabla e_{\alpha}, \psi_{\alpha}\right\rangle_{\tilde{g}} d v_{\tilde{g}} \\
& \lesssim\left\|\sqrt{\tilde{g}} \tilde{g}^{i j} \nabla_{i} e_{\alpha} \psi_{\alpha}^{j}\right\|_{\mathscr{H} \mathcal{H}^{1}}\left[\left(u-u_{2 \theta}\right) \eta\right]_{B M O} \\
& \lesssim\left\|\psi_{\alpha}\right\|_{L^{2}\left(B_{2 \theta}\right)}\left\|\nabla e_{\alpha}\right\|_{L^{2}\left(B_{2 \theta}\right)}\left[\left(u-u_{2 \theta}\right) \eta\right]_{B M O} \\
& \lesssim\|\nabla u\|_{L^{2}\left(B_{2 \theta}\right)}\left\|\psi_{\alpha}\right\|_{L^{2}\left(B_{2 \theta}\right)}\|\nabla u\|_{M^{p, p}\left(B_{1}\right)},
\end{aligned}
$$


where we have used the inequality

$$
\left[\left(u-u_{2 \theta}\right) \eta\right]_{B M O} \leq C[u]_{B M O\left(B_{2 \theta}\right)} \leq C\|\nabla u\|_{M^{p, p}\left(B_{1}\right)} .
$$

This, combined with Hölder's inequality, implies

$$
\left(\theta^{p-n} \int_{B_{\theta}}\left|\psi_{\alpha}\right|^{p}\right)^{1 / p} \leq C \epsilon_{0}\|\nabla u\|_{M^{p, p}\left(B_{1}\right)} .
$$

Step 5: Decay estimation of $\nabla u$. Putting (4-12) and (4-13) together, we have that, for some $0<\alpha_{0}<1$,

$$
\left((\tau \theta)^{p-n} \int_{B_{\tau \theta}}|\nabla u|^{p}\right)^{1 / p} \leq C\left(\epsilon_{0}+\tau^{\alpha_{0}}+\tau^{1-n / p} \epsilon_{0}\right)\|\nabla u\|_{M^{p, p}\left(B_{1}\right)}
$$

for any $0<\tau<1$ and $0<\theta<\frac{1}{2}$. Now we claim that for some $\alpha_{0} \in(0,1)$, we have

$$
\|\nabla u\|_{M^{p, p}\left(B_{\tau / 4}\right)} \leq C\left(\epsilon_{0}+\tau^{\alpha_{0}}+\tau^{1-n / p} \epsilon_{0}\right)\|\nabla u\|_{M^{p, n-p}\left(B_{1}\right)}
$$

for all $0<\tau<1$. To show this, let $B_{s}(y) \subset B_{\tau / 4}$. We divide into three cases:

(a) $y \in B_{\tau / 4} \cap B^{ \pm}$and $s<\left|y^{n}\right|$. As remarked at the beginning of the proof, for some $0<\alpha_{0}<1$ we have

$$
\begin{aligned}
\left(s^{p-n} \int_{B_{s}(y)}|\nabla u|^{p}\right)^{1 / p} & \leq C\left(\frac{s}{\left|y^{n}\right|}\right)^{\alpha_{0}}\left(\left|y^{n}\right|^{p-n} \int_{B_{\left|y^{n}\right|}(y)}|\nabla u|^{p}\right)^{1 / p} \\
& \leq C\left(\frac{s}{\left|y^{n}\right|}\right)^{\alpha_{0}}\left(\left(2\left|y^{n}\right|\right)^{p-n} \int_{B_{2\left|y^{n}\right|}\left(y^{\prime}, 0\right)}|\nabla u|^{p}\right)^{1 / p} \\
& \leq C\left(\left(\frac{\tau}{2}\right)^{p-n} \int_{B_{\tau / 2}\left(y^{\prime}, 0\right)}|\nabla u|^{p}\right)^{1 / p}\left(\text { since }\left|y^{n}\right| \leq \tau / 4\right) \\
& \leq C\left(\epsilon_{0}+\tau^{\alpha_{0}}+\tau^{1-n / p} \epsilon_{0}\right)\|\nabla u\|_{M^{p, p}\left(B_{1}\right)} \text { (by (4-14)). }
\end{aligned}
$$

(b) $y \in B_{\tau / 4} \cap B^{ \pm}$and $s \geq\left|y^{n}\right|$. Then $B_{s}(y) \subset B_{\left|y^{n}\right|+s}\left(y^{\prime}, 0\right) \subset B_{2 s}\left(y^{\prime}, 0\right)$. Hence

$$
\begin{aligned}
\left(s^{p-n} \int_{B_{s}(y)}|\nabla u|^{p}\right)^{1 / p} & \leq 2^{n / p-1}\left((2 s)^{p-n} \int_{B_{2 s}\left(y^{\prime}, 0\right)}|\nabla u|^{p}\right)^{1 / p} \\
& \leq C\left(\epsilon_{0}+\tau^{\alpha_{0}}+\tau^{1-n / p} \epsilon_{0}\right)\|\nabla u\|_{M^{p, p}\left(B_{1}\right)} \text { (by (4-14)). }
\end{aligned}
$$

(c) $y \in B_{\tau / 4} \cap \Gamma_{1}$, i.e., $y^{n}=0$. Then it follows directly from (4-14) that

$$
\left(s^{p-n} \int_{B_{s}(y)}|\nabla u|^{p}\right)^{1 / p} \leq C\left(\epsilon_{0}+\tau^{\alpha_{0}}+\tau^{1-n / p} \epsilon_{0}\right)\|\nabla u\|_{M^{p, p}\left(B_{1}\right)} .
$$

Combining (a), (b) and (c) together and taking the supremum over all $B_{s}(y) \subset B_{\tau / 4}$, we obtain (4-15). 
It is clear that by first choosing $\tau$ and then $\epsilon$ sufficiently small, we can arrange that

$$
\|\nabla u\|_{M^{p, p}\left(B_{\tau / 4}\right)} \leq \frac{1}{2}\|\nabla u\|_{M^{p, p}\left(B_{1}\right)} .
$$

Iterating this inequality finitely many times yields that there exists $\alpha_{1} \in(0,1)$ such that for any $x \in B_{1 / 4}$ and $0<r \leq \frac{1}{2}$, it holds

$$
r^{p-n} \int_{B_{r}(x)}|\nabla u|^{p} d x \leq C r^{p \alpha_{1}}\|\nabla u\|_{M^{p, p}\left(B_{1}\right)}^{p} .
$$

This implies $u \in C^{\alpha_{1}}\left(B_{1 / 2}\right)$ by Morrey's lemma. The proof is now completed.

\section{Lipschitz and piecewise $C^{1, \alpha}$-regularity}

In this section, we will first establish Lipschitz and piecewise $C^{1, \alpha}$-regularity for stationary harmonic maps on domains with piecewise $C^{0,1}$-metrics, under a smallness condition of energy. Then we will prove Theorem 1.1.

Theorem 5.1. There exist $\epsilon_{0}>0$ and $\beta_{0} \in(0,1)$, depending only on $n$ and $g$, such that if the metric $g \in C^{0,1}\left(B_{1}^{ \pm} \cup \Gamma_{1}\right)$ satisfies the condition (1-4) on $\Gamma_{1}$, and $u \in W^{1,2}\left(B_{1}, N\right)$ is a stationary harmonic map on $\left(B_{1}, g\right)$ satisfying

$$
r_{0}^{2-n} \int_{B_{r_{0}}\left(x_{0}\right)}|\nabla u|_{g}^{2} d v_{g} \leq \epsilon_{0}^{2}
$$

for some $x_{0} \in B_{1 / 2}$ and $0<r_{0} \leq \frac{1}{4}$, then $u \in C^{1, \beta_{0}}\left(B_{r_{0} / 2}\left(x_{0}\right) \cap \overline{B^{ \pm}}, N\right)$, and $u \in C^{0,1}\left(B_{r_{0} / 2}\left(x_{0}\right), N\right)$.

Proof. The proof is based on the hole filling argument and the freezing coefficient method. It is divided into two steps.

Step 1: $u \in C^{\alpha}\left(B_{3 r_{0} / 4}\left(x_{0}\right), N\right)$ for any $0<\alpha<1$. To see this, first recall Theorem 4.1 implies that there exists $0<\alpha_{0}<\frac{2}{3}$ such that $u \in C^{\alpha_{0}}\left(B_{7 r_{0} / 8}\left(x_{0}\right)\right)$ and for any $y \in B_{7 r_{0} / 8}\left(x_{0}\right)$, it holds

$$
s^{2-n} \int_{B_{s}(y)}|\nabla u|^{2} d x \leq C\left(\frac{s}{r}\right)^{2 \alpha_{0}} r^{2-n} \int_{B_{r}(y)}|\nabla u|^{2} d x, \quad 0<s \leq r<\frac{r_{0}}{8}
$$

and

$$
\operatorname{osc}_{B_{r}(y)} u \leq C r^{\alpha_{0}}, \quad 0<r<\frac{r_{0}}{8} .
$$

For $y \in B_{7 r_{0} / 8}\left(x_{0}\right)$ and $0<r<r_{0} / 8$, let $v: B_{r}(y) \rightarrow \mathbb{R}^{k}$ solve

$$
\begin{cases}\Delta_{g} v=0 & \text { in } B_{r}(y) \\ v=u & \text { on } \partial B_{r}(y) .\end{cases}
$$


By the maximum principle and (5-3), we then have

$$
\operatorname{Osc}_{B_{r}(y)} v \leq \operatorname{osc}_{\partial B_{r}(y)} u \leq C r^{\alpha_{0}} .
$$

Moreover, since $g \in C^{0,1}\left(B_{1}^{ \pm} \cup \Gamma_{1}\right)$, it follows from [Li and Nirenberg 2003, Theorem 1.1] that $v \in C^{0,1}\left(B_{r / 2}(y), \mathbb{R}^{k}\right)$ and $v \in C^{1, \beta}\left(B_{r / 2}(y) \cap \overline{B^{ \pm}}, \mathbb{R}^{k}\right)$ for any $0<\beta<1$.

Multiplying (1-1) and (5-4) by $u-v$, subtracting one result from the other and integrating over $B_{r}(y)$, we obtain

$$
\int_{B_{r}(y)}|\nabla(u-v)|^{2} d x \lesssim \int_{B_{r}(y)}|\nabla u|^{2}|u-v| \lesssim r^{n-2+3 \alpha_{0}} .
$$

This, combined with

$$
\int_{B_{r / 2}(y)}|\nabla v|^{2} d x \leq C\|\nabla v\|_{L^{\infty}\left(B_{r / 2}(y)\right)}^{2} r^{n},
$$

implies

$$
\left(\frac{r}{2}\right)^{2-n} \int_{B_{r / 2}(y)}|\nabla u|^{2} d x \leq C\left(\|\nabla v\|_{L^{\infty}\left(B_{r / 2}(y)\right)}^{2} r^{2}+r^{3 \alpha_{0}}\right) \leq C r^{3 \alpha_{0}} .
$$

This, combined with Morrey's lemma, yields $u \in C^{3 \alpha_{0} / 2}\left(B_{7 r_{0} / 8}\left(x_{0}\right)\right)$. Repeating this argument, we can show that $u \in C^{\alpha}\left(B_{3 r_{0} / 4}\left(x_{0}\right)\right)$ for any $0<\alpha<1$, and

$$
r^{2-n} \int_{B_{r}(y)}|\nabla u|^{2} d x \leq C r^{2 \alpha} \quad \text { for all } y \in B_{3 r_{0} / 4}\left(x_{0}\right), 0<r<\frac{r_{0}}{4} .
$$

Step 2: There exists $0<\beta_{0}<1$ such that $u \in C^{1, \beta_{0}}\left(B_{r_{0} / 2}\left(x_{0}\right) \cap \overline{B^{ \pm}}, N\right)$. There are two cases to consider:

Case I: $x_{0}=\left(x_{0}^{\prime}, x_{0}^{n}\right) \in B_{1}^{ \pm}$. We may assume $0<r_{0}<\left|x_{0}^{n}\right|$, so that $B_{r_{0}}\left(x_{0}\right) \subset B^{ \pm}$. For $B_{r}(x) \subset B_{r_{0}}\left(x_{0}\right)$, let $v: B_{r}(x) \rightarrow \mathbb{R}^{k}$ solve

$$
\begin{cases}\Delta_{g} v=0 & \text { in } B_{r}(x) \\ v=u & \text { on } \partial B_{r}(x)\end{cases}
$$

Then by (5-5), for any $\frac{2}{3}<\alpha<1$,

$$
\int_{B_{r}(x)}|\nabla(u-v)|^{2} d x \leq C \int_{B_{r}(x)}|\nabla u|^{2}|u-v| d x \leq C r^{3 \alpha+n-2} .
$$

Also, since $g \in C^{0,1}\left(B_{r_{0}}\left(x_{0}\right)\right)$, we have for any $0<\beta<1$ that $v \in C^{1, \beta}\left(B_{r / 2}(x)\right)$ and

$$
f_{B_{s}(x)}\left|\nabla v-(\nabla v)_{B_{s}(x)}\right|^{2} d x \leq C\left(\frac{s}{r}\right)^{2 \beta} f_{B_{r}(x)}\left|\nabla u-(\nabla u)_{B_{r}(x)}\right|^{2} d x,
$$


for $<s \leq r / 2$. (Here $f_{E} f=\frac{1}{|E|} \int_{E} f d x$.) Note that (5-8) also holds trivially for $r / 2 \leq s \leq r$. Combining (5-7) and (5-8) we obtain, for any $0<\theta<1$,

$$
\begin{aligned}
f_{B_{\theta r}(x)}\left|\nabla u-(\nabla u)_{B_{\theta r}(x)}\right|^{2} d x & \\
& \leq 2\left(f_{B_{\theta r}(x)}|\nabla u-\nabla v|^{2} d x+f_{B_{\theta r}(x)}\left|\nabla v-(\nabla v)_{B_{\theta r}(x)}\right|^{2} d x\right) \\
& \leq C\left(\theta^{2 \beta} f_{B_{r}(x)}\left|\nabla u-(\nabla u)_{B_{r}(x)}\right|^{2} d x+\theta^{-n} r^{3 \alpha-2}\right) .
\end{aligned}
$$

For $(3 \alpha-2) / 2<\beta_{0}<\beta$, let $0<\theta_{0}<1$ be such that $C \theta_{0}^{2 \beta}=\theta_{0}^{2 \beta_{0}}$. Then

$$
f_{B_{\theta_{0}}(x)}\left|\nabla u-(\nabla u)_{B_{\theta_{0}}(x)}\right|^{2} d x \leq \theta_{0}^{2 \beta_{0}} f_{B_{r}(x)}\left|\nabla u-(\nabla u)_{B_{r}(x)}\right|^{2} d x+C r^{3 \alpha-2}
$$

Iterating (5-9) $m$-times, $m \geq 1$, yields

$$
\begin{aligned}
& f_{B_{\theta_{0}^{m}}(x)}\left|\nabla u-(\nabla u)_{B_{\theta_{0}}(x)}\right|^{2} d x \\
& \leq\left(\theta_{0}^{m}\right)^{2 \beta_{0}} f_{B_{r}(x)}\left|\nabla u-(\nabla u)_{B_{r}(x)}\right|^{2} d x+C\left(\theta_{0}^{m} r\right)^{3 \alpha-2} \sum_{j=1}^{m} \theta_{0}^{j\left(2 \beta_{0}-(3 \alpha-2)\right)} \\
& \leq\left(\theta_{0}^{m}\right)^{3 \alpha-2}\left(f_{B_{r}(x)}\left|\nabla u-(\nabla u)_{B_{r}(x)}\right|^{2} d x+C r^{3 \alpha-2}\right)
\end{aligned}
$$

This clearly implies that $\nabla u \in C^{3 \alpha / 2-1}\left(B_{r_{0}}\left(x_{0}\right)\right)$.

Case II: $x_{0}=\left(x_{0}^{\prime}, 0\right) \in \Gamma_{1}$. For simplicity, we assume $x_{0}^{\prime}=0$. Define $\bar{g}$ on $B_{1}$ by

$$
\bar{g}(x)=\left\{\begin{array}{ll}
\lim _{t \downarrow 0^{+}} g\left(0^{\prime}, t\right) & \text { if } x \in B_{1}^{+} \\
\lim _{t \uparrow 0^{-}} g\left(0^{\prime}, t\right) & \text { if } x \in B_{1}^{-}
\end{array} .\right.
$$

Then we have

$$
|g(x)-\bar{g}(x)| \leq C|x|, \quad x \in B_{1} .
$$

Moreover, by suitable dilations and rotations of the coordinate system, (1-4) implies that there exists a positive constant $k \neq 1$ such that

$$
\bar{g}(x)=\left(1+(k-1) \chi_{B_{1}^{-}}(x)\right) g_{0}, \quad x \in B_{1},
$$

where $\chi_{B_{1}^{-}}$is the characteristic function of $B_{1}^{-}$.

For $0<r<r_{0} / 2$, let $v: B_{r}(0) \rightarrow \mathbb{R}^{k}$ solve

$$
\begin{cases}\Delta_{\bar{g}} v=0 & \text { in } B_{r}(0) \\ v=u & \text { on } \partial B_{r}(0) .\end{cases}
$$


Then we have

$$
\operatorname{osc}_{B_{r}(0)} v \leq \operatorname{osc}_{B_{r}(0)} u \leq C r^{\alpha}, \quad \int_{B_{r}(0)}|\nabla v|^{2} d x \leq C \int_{B_{r}(0)}|\nabla u|^{2} \leq C r^{n-2+2 \alpha} .
$$

Multiplying (1-1) and (5-12) by $u-v$ and integrating over $B_{r}(0)$, we obtain

$$
\begin{aligned}
\int_{B_{r}(0)}|\nabla(u-v)|^{2} d x \\
\quad \leq \int_{B_{r}(0)} g^{i j}(u-v)_{i}(u-v)_{j} \sqrt{g} d x \\
\leq C \int_{B_{r}(0)}|\nabla u|^{2}|u-v| d x+\int_{B_{r}(0)}\left|\sqrt{g} g^{i j}-\sqrt{\bar{g}} \bar{g}^{i j}\right|\left|v_{i}\right|\left|(u-v)_{j}\right| d x \\
\quad \leq C \operatorname{osc}_{B_{r}(0)} v \int_{B_{r}(0)}|\nabla u|^{2} d x+C r^{2} \int_{B_{r}(0)}|\nabla v|^{2}+\frac{1}{2} \int_{B_{r}(0)}|\nabla(u-v)|^{2} d x \\
\leq C r^{n-2+3 \alpha}+C r^{n+\alpha}+\frac{1}{2} \int_{B_{r}(0)}|\nabla(u-v)|^{2} d x .
\end{aligned}
$$

This implies

$$
\int_{B_{r}(0)}|\nabla(u-v)|^{2} d x \leq C r^{n-2+3 \alpha} .
$$

It is well-known that $v \in C^{\infty}\left(\overline{B_{s}^{ \pm}(0)}\right)$ for any $0<s<r$. In fact, (5-12) is equivalent to:

$$
\frac{\partial}{\partial x_{i}}\left(\left(1+\left(k^{n / 2}-1\right) \chi_{B_{1}^{-}}\right) \frac{\partial v}{\partial x_{i}}\right)=0 \quad \text { in } B_{r}(0)
$$

we conclude

(i) $\partial v / \partial x_{n}$ satisfies the jump property on $\Gamma_{1}$ :

$$
\lim _{x_{n} \downarrow 0^{+}} \frac{\partial v}{\partial x_{n}}\left(x^{\prime}, x_{n}\right)=k^{n / 2} \lim _{x_{n} \uparrow 0^{-}} \frac{\partial v}{\partial x_{n}}\left(x^{\prime}, x_{n}\right) \quad \text { for all }\left(x^{\prime}, 0\right) \in \Gamma_{1} \cap B_{r}(0) .
$$

(ii) $\nabla^{\alpha} v \in C^{0}\left(B_{r}(0)\right)$ for any multiindex $\alpha=\left(\alpha_{1}, \ldots, \alpha_{n-1}, 0\right)$.

(iii) $\nabla v \in L^{\infty}\left(B_{s}(0)\right)$ for any $0<s<r$, and

$$
\|\nabla v\|_{L^{\infty}\left(B_{r / 2}(0)\right)}^{2} \leq C r^{2-n} \int_{B_{r}(0)}|\nabla u|^{2} .
$$

For $f: B_{r}(0) \rightarrow \mathbb{R}^{k}$, set

$$
\widetilde{D} f:=\left(\frac{\partial f}{\partial x_{1}}, \ldots, \frac{\partial f}{\partial x_{n-1}},\left(1+\left(k^{n / 2}-1\right) \chi_{B_{1}^{-}}\right) \frac{\partial f}{\partial x_{n}}\right),
$$


and denote by $(\widetilde{D} f)_{s}=f_{B_{s}(0)} \widetilde{D} f d x$ the average of $\widetilde{D} f$ over $B_{s}(0)$. Then, for any $0<\beta<1$,

$$
f_{B_{s}(0)}\left|\widetilde{D} v-(\widetilde{D} v)_{s}\right|^{2} d x \leq C\left(\frac{s}{r}\right)^{2 \beta} f_{B_{r}(0)}\left|\widetilde{D} u-(\widetilde{D} u)_{r}\right|^{2} d x \quad \text { for all } 0<s \leq r .
$$

Combining this with (5-13) yields, for any $0<\theta<1$,

$$
f_{B_{\theta r}(0)}\left|\widetilde{D} u-(\widetilde{D} u)_{\theta r}\right|^{2} d x \leq C \theta^{2 \beta} f_{B_{r}(0)}\left|\widetilde{D} u-(\widetilde{D} u)_{r}\right|^{2} d x+C \theta^{-n} r^{3 \alpha-2}
$$

As in case I, iterations of this inequality yield, for any $0<s \leq r$,

$$
f_{B_{s}(0)}\left|\widetilde{D} u-(\widetilde{D} u)_{s}\right|^{2} d x \leq C\left(\frac{s}{r}\right)^{3 \alpha-2} f_{B_{r}(0)}\left|\widetilde{D} u-(\widetilde{D} u)_{r}\right|^{2} d x+C s^{3 \alpha-2} .
$$

This, combined with case I, implies that for any $B_{r}(x) \subset B_{r_{0}}\left(x_{0}\right)$ and $0<s \leq r$,

$$
f_{B_{s}(x)}\left|\widetilde{D} u-(\widetilde{D} u)_{x, s}\right|^{2} d x \leq C\left(\frac{s}{r}\right)^{3 \alpha-2} f_{B_{r}(x)}\left|\widetilde{D} u-(\widetilde{D} u)_{x, r}\right|^{2} d x+C s^{3 \alpha-2},
$$

where $(\widetilde{D} u)_{x, s}$ denotes the average of $\widetilde{D} u$ over $B_{S}(x)$. It is readily seen that the preceding inequality yields $u \in C^{1,3 \alpha / 2-1}\left(B_{r_{0} / 2}\left(x_{0}\right) \cap \overline{B_{1}^{ \pm}}\right)$and $u \in C^{0,1}\left(B_{r_{0} / 2}\left(x_{0}\right)\right)$. This completes the proof.

Proof of Theorem 1.1. Define the singular set

$$
\Sigma=\left\{x \in \Omega: \varliminf_{r \rightarrow 0} r^{2-n} \int_{B_{r}(x)}|\nabla u|^{2} d x \geq \epsilon_{0}^{2}\right\} .
$$

Then by a covering argument we have $H^{n-2}(\Sigma)=0$; see [Evans and Gariepy 1992]. For any $x_{0} \in \Omega \backslash \Sigma$, there exists $0<r_{0}<\operatorname{dist}\left(x_{0}, \partial \Omega\right)$ such that

$$
r_{0}^{2-n} \int_{B_{r_{0}}(x)}|\nabla u|^{2} d x \leq \epsilon_{0}^{2} .
$$

Hence by Theorems 2.1, 4.1, and 5.1, we have

$$
u \in C^{1, \alpha}\left(B_{r_{0} / 2}\left(x_{0}\right) \cap \overline{\Omega^{ \pm}}, N\right) \quad \text { and } \quad u \in C^{0,1}\left(B_{r_{0} / 2}\left(x_{0}\right), N\right),
$$

for some $0<\alpha<1$. In particular, we have

$$
\lim _{r \downarrow 0} r^{2-n} \int_{B_{r}(x)}|\nabla u|^{2} d x=0 \text { for all } x \in B_{r_{0} / 2}\left(x_{0}\right),
$$

so that $B_{r_{0} / 2}\left(x_{0}\right) \cap \Sigma=\varnothing$, i.e., $\Sigma$ is closed. This completes the proof. 


\section{Harmonic maps to manifolds supporting convex distance square functions}

In this section, we consider weakly harmonic maps $u$ from $(\Omega, g)$, with $g$ the piecewise Lipschitz continuous metric as in Theorem 1.1, to $(N, h)$, whose universal cover $(\tilde{N}, \tilde{h})$ supports a convex distance square function $d_{\widetilde{N}}^{2}(\cdot, p)$ for any $p \in \tilde{N}$. We will establish both the global Lipschitz continuity and piecewise $C^{1, \alpha}$-regularity for such harmonic maps $u$. This can be viewed as a generalization of the well-known regularity theorem of Eells and Sampson [1964] and Hildebrand, Kaul and Widman [Hildebrandt et al. 1977].

The crucial step is the following theorem on Hölder continuity.

Theorem 6.1. Assume that the metric $g$ is bounded measurable on $\Omega$, i.e., there exist two constants $0<\lambda<\Lambda<+\infty$ such that $\lambda \rrbracket_{n} \leq g(x) \leq \Lambda \rrbracket_{n}$ for a.e. $x \in \Omega$, and the universal cover $(\tilde{N}, \tilde{h})$ of $(N, h)$ supports a convex distance square function $d_{\widetilde{N}}^{2}(\cdot, p)$ for any $p \in \widetilde{N}$. If $u \in H^{1}(\Omega, N)$ is a weakly harmonic map, then there exists $\alpha \in(0,1)$ such that $u \in C^{\alpha}(\Omega, N)$.

Proof. Here we sketch a proof that is based on modifications of that in [Lin 1997]. Similar ideas have been used by Evans in his celebrated work [1982] and by Caffarelli [1982] for quasilinear systems under smallness conditions. First, by lifting $u: \Omega \rightarrow N$ to a harmonic map $\tilde{u}: \Omega \rightarrow \tilde{N}$, we may assume $(N, h)=(\tilde{N}, \tilde{h})$ and $d_{N}^{2}(\cdot, p)$ is convex on $N$ for any $p \in N$.

We first claim that

$$
\Delta_{g} d^{2}(u, p) \geq 0
$$

In fact, by the chain rule of harmonic maps (see [Jost 1991]), we have

$$
\Delta_{g} d^{2}(u, p)=\nabla_{u} d^{2}(u, p)\left(\Delta_{g} u\right)+\nabla_{u}^{2} d^{2}(u, p)(\nabla u, \nabla u)_{g} .
$$

Since $\Delta_{g} u \perp T_{u} N, \nabla_{u} d^{2}(u, p) \in T_{u} N$, the first term in the right side vanishes. By the convexity of $d_{N}^{2}$, the second term in the right side satisfies

$$
\nabla_{u}^{2} d^{2}(u, p)(\nabla u, \nabla u)_{g} \geq 0 .
$$

Since $u \in H^{1}(\Omega, N)$, by suitably choosing $p \in N$ and applying Poincaré inequality and Harnack's inequality, (6-1) implies $u \in L_{\mathrm{loc}}^{\infty}(\Omega, N)$.

For a set $E \subset N$, let $\operatorname{diam}_{N} E$ denote the diameter of $E$ with respect to the distance function $d_{N}(\cdot, \cdot)$. For any ball $B_{r}(x) \subset \Omega$, we want to show that $u \in C^{\alpha}\left(B_{r / 2}(x)\right)$ for some $0<\alpha<1$. To do it, set $C_{r}:=\operatorname{diam}_{N} u\left(B_{r}(x)\right)$. We may assume $C_{r}>0$ (otherwise, $u$ is constant on $B_{r}(x)$ and we are done). Now we want to show that there exists $0<\delta_{0}=\delta_{0}(N) \leq \frac{1}{2}$ such that

$$
\operatorname{diam}_{N} u\left(B_{\delta_{0} r}(x)\right) \leq \frac{1}{2} C_{r} .
$$


Since $u_{r}(y)=u(x+r y): B_{1}(0) \rightarrow N$ is a harmonic map $\left(B_{1}(0), g_{r}\right)$, with $g_{r}(y)=$ $g(x+r y)$, we may, for simplicity, assume $x=0$ and $r=2$. For any $0<\epsilon<\frac{1}{2}$, since $u\left(B_{1}\right) \subset N$ is a bounded set, there exists $m=m(\epsilon) \geq 1$ such that $u\left(B_{1}\right)$ is covered by $m$ balls $B^{1}, \ldots, B^{m}$ of radius $\epsilon C_{1}$.

Claim. There exists sufficiently small $\epsilon>0$ such that $u\left(B_{1 / 2}\right)$ can be covered by at most $(m-1)$ balls among $B^{1}, \ldots, B^{m}$.

To see this, let $x_{i} \in B_{1}$ such that $B^{i} \subset B_{2 \epsilon C_{1}}\left(p_{i}\right), p_{i}=u\left(x_{i}\right)$, for $1 \leq i \leq m$. Let $1 \leq m^{\prime} \leq m$ be the maximum number of points in $\left\{p_{i}\right\}_{i=1}^{m}$ such that the distance between any two of them is at least $C_{1} / 32$. Thus the sets $B_{C_{1} / 16}\left(p_{i}\right)$, for $1 \leq i \leq$ $m^{\prime}$, cover $u\left(B_{1}\right)$. For convenience, set $U_{i}=u^{-1}\left(B^{N}\left(p_{i}, C_{1} / 16\right)\right)$, the notation $B^{N}(x, R)$ referring to the ball in $N$ with center $x$ and radius $R$. We will show that there exists $i_{0} \in\left\{1, \ldots, m^{\prime}\right\}$ such that

$$
\frac{1}{4} C_{1}^{2} \leq \sup _{x \in B_{2}} d_{N}^{2}\left(u(x), p_{i_{0}}\right) \leq C_{1}^{2},
$$

and

$$
H^{n}\left(U_{i_{0}} \cap B_{1 / 2}\right) \geq c_{0},
$$

for some universal constant $c_{0}>0$. Indeed, since $B_{1 / 2} \subset \bigcup_{i=1}^{m^{\prime}} U_{i}$, we have

$$
\sum_{i=1}^{m^{\prime}} H^{n}\left(U_{i} \cap B_{1 / 2}\right) \geq H^{n}\left(B_{1 / 2}\right) .
$$

Hence there exists $i_{0} \in\left\{1, \ldots, m^{\prime}\right\}$ such that

$$
H^{n}\left(U_{i} \cap B_{1 / 2}\right) \geq c_{0}:=\frac{1}{m^{\prime}} H^{n}\left(B_{1 / 2}\right) .
$$

This implies (6-4). Now (6-3) follows from the triangle inequality.

Next we define

$$
f(x):=\sup _{z \in B_{1}} d_{N}^{2}\left(u(z), p_{i_{0}}\right)-d_{N}^{2}\left(u(x), p_{i_{0}}\right), \quad x \in B_{1} .
$$

It is clear that $f \geq 0$ in $B_{1}$, and (6-1) implies $\Delta_{g} f \leq 0$ in $B_{1}$. By Moser's Harnack inequality, we have

$$
\begin{aligned}
\inf _{B_{1 / 2}} f & \geq C f_{B_{1}} f \geq C \int_{B_{1 / 2}} f \geq C \int_{B_{1 / 2} \cap U_{i_{0}}} f \\
& \geq C\left(\sup _{B_{1}} d_{N}^{2}\left(u, p_{i_{0}}\right)-\sup _{B_{1} \cap U_{i_{0}}} d_{N}^{2}\left(u, p_{i_{0}}\right)\right) H^{n}\left(B_{1 / 2} U_{i_{0}}\right) \\
& \geq C\left(\frac{1}{4} C_{1}^{2}-\frac{1}{256} C_{1}^{2}\right) c_{0}=: \theta_{0}^{2} C_{1}^{2}
\end{aligned}
$$


for some universal constant $\theta_{0}>0$. This implies

$$
\sup _{z \in B_{1}} d_{N}\left(u(z), p_{i_{0}}\right)-\sup _{z \in B_{1 / 2}} d_{N}\left(u(z), p_{i_{0}}\right) \geq \theta_{0} C_{1}=\left(1-\theta_{0}\right) C_{1} .
$$

Now we argue that the claim follows from (6-5). For, otherwise, we would have $u\left(B_{1 / 2}\right) \cap B_{2 \epsilon C_{1}}\left(p_{j}\right) \neq \varnothing$ for all $1 \leq j \leq m$. Let $z_{0} \in B_{1}$ be such that

$$
\epsilon C_{1}+d_{N}\left(u\left(z_{0}\right), p_{i_{0}}\right) \geq \sup _{B_{1}} d_{N}\left(u(z), p_{i_{0}}\right) .
$$

Since $u\left(B_{1}\right) \subset \bigcup_{i=}^{m} B_{2 \epsilon C_{1}}\left(p_{i}\right)$, there exists $p_{i_{1}} \in\left\{p_{1}, \ldots, p_{m}\right\}$ such that $u\left(z_{0}\right) \in$ $B_{2 \epsilon C_{1}}\left(p_{i_{1}}\right)$. Since $u\left(B_{1 / 2}\right) \cap B_{2 \epsilon C_{1}}\left(p_{i_{1}}\right) \neq \varnothing$, there exists $z_{1} \in B_{1 / 2}$ such that $u\left(z_{1}\right) \in B_{2 \epsilon C_{1}}\left(p_{i_{1}}\right)$. Therefore we have $d_{N}\left(u\left(z_{1}\right), u\left(z_{0}\right)\right) \leq 2 \epsilon C_{1}$. Therefore we have

$$
\begin{aligned}
\sup _{z \in B_{1}} d_{N}\left(u(z), p_{i_{0}}\right)-\sup _{z \in B_{1 / 2}} d_{N}\left(u(z), p_{i_{0}}\right) & \leq \epsilon C_{1}+d_{N}\left(u\left(z_{0}\right), p_{i_{0}}\right)-d_{N}\left(u\left(z_{1}\right), p_{i_{0}}\right) \\
& \leq \epsilon C_{1}+d_{N}\left(u\left(z_{0}\right), u\left(z_{1}\right)\right) \leq 3 \epsilon C_{1} .
\end{aligned}
$$

This contradicts (6-5) if $\epsilon>0$ is chosen to be sufficiently small.

From this claim, we have either

(i) $\operatorname{diam}_{N} u\left(B_{1 / 2}\right) \leq \frac{1}{2} C_{1}$-in which case (6-2) holds with $\delta_{0}=\frac{1}{2}-$ or

(ii) $\operatorname{diam}_{N} u\left(B_{1 / 2}\right)>\frac{1}{2} C_{1}$.

Then we consider $v(x)=u(x / 2): B_{1} \rightarrow N$ and conclude:

- $v$ is a harmonic map on $\left(B_{1}, g_{1 / 2}\right)$, with the metric $g_{1 / 2}(x)=g(x / 2)$.

- $\frac{1}{2} C_{1}<\operatorname{diam}_{N} v\left(B_{1}\right) \leq C_{1}$.

- $v\left(B_{1}\right)$ is covered by at most $m-1$ balls $B_{1}, \ldots, B^{m-1}$ of radius $\epsilon C_{1}$.

Thus the claim is applicable to $v$ so that $u\left(B_{1 / 4}\right)=v\left(B_{1 / 2}\right)$ can be covered by at most $m-2$ balls among $B^{1}, \ldots, B^{m-1}$.

If $\operatorname{diam}_{N} v\left(B_{1 / 2}\right) \leq \frac{1}{2} C_{1}$, we are done. Otherwise, we can repeat the above argument. It is clear that the process can at most be repeated $m$ times, and the process will not be stopped at step $k_{0} \leq m$ unless $\operatorname{diam}_{N} u\left(B_{2^{-k_{0}}}\right) \leq \frac{1}{2} C_{1}$. Thus (6-2) is proven.

It is readily seen that iteration of (6-2) implies Hölder continuity.

Proof of Theorem 1.2. First, by Theorem 6.1 and the argument from Section 4, we can show that for some $0<\alpha<1$,

$$
\int_{B_{r}(x)}|\nabla u|^{2} d x \leq C r^{n-2+2 \alpha} \text { for all } B_{r}(x) \subset \Omega .
$$

Then we can follow the proof of (5-2) to show that $u \in C^{0,1}(\Omega) \cap C^{1, \alpha}\left(\Omega^{ \pm} \cup \Gamma, N\right)$. 


\section{Acknowledgements}

Part of this work was completed while Li visited the University of Kentucky. He thanks the Department of Mathematics for its hospitality. Li was partially supported by SRFDPHE (20100003120005) and NSFC in China (11071020, 11126038) and Program for Changjiang Scholars and Innovative Research Team in University in China (IRT0908). Wang is partially supported by NSF grant 1000115.

\section{References}

[Avellaneda and Lin 1987] M. Avellaneda and F.-H. Lin, "Compactness methods in the theory of homogenization”, Comm. Pure Appl. Math. 40:6 (1987), 803-847. MR 88i:35019 Zbl 0632.35018

[Bethuel 1993] F. Bethuel, "On the singular set of stationary harmonic maps", Manuscripta Math. 78:4 (1993), 417-443. MR 94a:58047 Zbl 0792.53039

[Byun and Wang 2010] S.-S. Byun and L. Wang, "Elliptic equations with measurable coefficients in Reifenberg domains", Adv. Math. 225:5 (2010), 2648-2673. MR 2012a:35046 Zbl 1198.35068

[Caffarelli 1982] L. A. Caffarelli, "Regularity theorems for weak solutions of some nonlinear systems", Comm. Pure Appl. Math. 35:6 (1982), 833-838. MR 83m:35052 Zbl 0496.35035

[Dong and Kim 2010] H. Dong and D. Kim, "Elliptic equations in divergence form with partially BMO coefficients", Arch. Ration. Mech. Anal. 196:1 (2010), 25-70. MR 2011b:35086 Zbl 1206.35249

[Dong and Kim 2011a] H. Dong and D. Kim, "Parabolic and elliptic systems in divergence form with variably partially BMO coefficients", SIAM J. Math. Anal. 43:3 (2011), 1075-1098. MR 2012h:35141 Zbl 1230.35045

[Dong and Kim 2011b] H. Dong and D. Kim, " $L_{p}$ solvability of divergence type parabolic and elliptic systems with partially BMO coefficients", Calc. Var. Partial Differential Equat. 40:3-4 (2011), 357-389. MR 2011m:35393 Zbl 1216.35160

[Eells and Sampson 1964] J. Eells, Jr. and J. H. Sampson, "Harmonic mappings of Riemannian manifolds”, Amer. J. Math. 86 (1964), 109-160. MR 29 \#1603 Zbl 0122.40102

[Evans 1982] L. C. Evans, "Classical solutions of fully nonlinear, convex, second-order elliptic equations”, Comm. Pure Appl. Math. 35:3 (1982), 333-363. MR 83g:35038 Zbl 0469.35022

[Evans 1991] L. C. Evans, "Partial regularity for stationary harmonic maps into spheres", Arch. Rational Mech. Anal. 116:2 (1991), 101-113. MR 93m:58026 Zbl 0754.58007

[Evans and Gariepy 1992] L. C. Evans and R. F. Gariepy, Measure theory and fine properties of functions, Studies in Advanced Mathematics, CRC Press, Boca Raton, FL, 1992. MR 93f:28001 Zbl 0804.28001

[Giaquinta and Hildebrandt 1982] M. Giaquinta and S. Hildebrandt, "A priori estimates for harmonic mappings", J. Reine Angew. Math. 336 (1982), 124-164. MR 84b:58035

[Gilbarg and Trudinger 1983] D. Gilbarg and N. S. Trudinger, Elliptic partial differential equations of second order, Second ed., Grundlehren der Mathematischen Wissenschaften [Fundamental Principles of Mathematical Sciences] 224, Springer, Berlin, 1983. MR 86c:35035 Zbl 0562.35001

[Hélein 2002] F. Hélein, Harmonic maps, conservation laws and moving frames, Second ed., Cambridge Tracts in Mathematics 150, Cambridge University Press, Cambridge, 2002. Translated from the 1996 French original, With a foreword by James Eells. MR 2003g:58024 Zbl 1010.58010 
[Hildebrandt et al. 1977] S. Hildebrandt, H. Kaul, and K.-O. Widman, "An existence theorem for harmonic mappings of Riemannian manifolds”, Acta Math. 138:1-2 (1977), 1-16. MR 55 \#6478 Zbl 0356.53015

[Ishizuka and Wang 2008] W. Ishizuka and C. Y. Wang, "Harmonic maps from manifolds of $L^{\infty}$ Riemannian metrics", Calc. Var. Partial Differential Equat. 32:3 (2008), 387-405. MR 2009f:35064 Zbl 1154.58005

[Jost 1991] J. Jost, Two-dimensional geometric variational problems, Pure and Applied Mathematics (New York), John Wiley \& Sons Ltd., Chichester, 1991. A Wiley-Interscience Publication. MR 92h:58045 Zbl 0729.49001

[Li and Nirenberg 2003] Y. Li and L. Nirenberg, "Estimates for elliptic systems from composite material", Comm. Pure Appl. Math. 56:7 (2003), 892-925. Dedicated to the memory of Jürgen K. Moser. MR 2004k:35097 Zbl 1125.35339

[Lin 1997] F. H. Lin, "Analysis on singular spaces", pp. 114-126 in Collection of papers on geometry, analysis and mathematical physics, World Sci. Publ., River Edge, NJ, 1997. MR 99j:53059 Zbl 1028.58019

[Lin and Yan 2003] F. Lin and X. Yan, "A type of homogenization problem", Discrete Contin. Dyn. Syst. 9:1 (2003), 1-30. MR 2003j:35021

[Rivière 1995] T. Rivière, "Everywhere discontinuous harmonic maps into spheres", Acta Math. 175:2 (1995), 197-226. MR 96k:58059 Zbl 0898.58011

Received March 7, 2012. Revised October 19, 2012.

\section{HAIGANG LI}

SChOol of Mathematical SCIEnCES AND Laboratory of Mathematics AND CompleX SYSTEMS, MiNisTRY OF EDUCATION

BEIJING NORMAL UNIVERSITY

BEIJING, 100875

CHINA

hgli@bnu.edu.cn

\section{CHANGYOU WANG}

DEPARTMENT OF MATHEMATICS

UNIVERSITY OF KENTUCKY

LEXINGTON, KY 40506

UNITED STATES

cywang@ms.uky.edu

http://www.ms.uky.edu/ cywang 


\title{
PACIFIC JOURNAL OF MATHEMATICS
}

\author{
msp.org/pjm
}

Founded in 1951 by E. F. Beckenbach (1906-1982) and F. Wolf (1904-1989)

\section{EDITORS}

V. S. Varadarajan (Managing Editor)

Department of Mathematics

University of California

Los Angeles, CA 90095-1555

pacific@math.ucla.edu

Paul Balmer

Department of Mathematics

University of California

Los Angeles, CA 90095-1555

balmer@math.ucla.edu

Daryl Cooper

Department of Mathematics

University of California

Santa Barbara, CA 93106-3080 cooper@math.ucsb.edu

Jiang-Hua $\mathrm{Lu}$

Department of Mathematics

The University of Hong Kong

Pokfulam Rd., Hong Kong jhlu@maths.hku.hk
Don Blasius

Department of Mathematics University of California

Los Angeles, CA 90095-1555

blasius@math.ucla.edu

Robert Finn

Department of Mathematics Stanford University

Stanford, CA 94305-2125

finn@math.stanford.edu

Sorin Popa

Department of Mathematics

University of California

Los Angeles, CA 90095-1555

popa@math.ucla.edu

Paul Yang

Department of Mathematics

Princeton University

Princeton NJ 08544-1000

yang@math.princeton.edu

\section{PRODUCTION}

Silvio Levy, Scientific Editor, production@msp.org

\section{SUPPORTING INSTITUTIONS}

ACADEMIA SINICA, TAIPEI

CALIFORNIA INST. OF TECHNOLOGY

INST. DE MATEMÁTICA PURA E APLICADA

KEIO UNIVERSITY

MATH. SCIENCES RESEARCH INSTITUTE

NEW MEXICO STATE UNIV.

OREGON STATE UNIV.

\author{
STANFORD UNIVERSITY \\ UNIV. OF BRITISH COLUMBIA \\ UNIV. OF CALIFORNIA, BERKELEY \\ UNIV. OF CALIFORNIA, DAVIS \\ UNIV. OF CALIFORNIA, LOS ANGELES \\ UNIV. OF CALIFORNIA, RIVERSIDE \\ UNIV. OF CALIFORNIA, SAN DIEGO \\ UNIV. OF CALIF., SANTA BARBARA
}

\author{
Vyjayanthi Chari \\ Department of Mathematics \\ University of California \\ Riverside, CA 92521-0135 \\ chari@math.ucr.edu \\ Kefeng Liu \\ Department of Mathematics \\ University of California \\ Los Angeles, CA 90095-1555 \\ liu@math.ucla.edu \\ Jie Qing \\ Department of Mathematics \\ University of California \\ Santa Cruz, CA 95064 \\ qing@cats.ucsc.edu
}

These supporting institutions contribute to the cost of publication of this Journal, but they are not owners or publishers and have no responsibility for its contents or policies.

See inside back cover or msp.org/pjm for submission instructions.

The subscription price for 2013 is US \$400/year for the electronic version, and \$485/year for print and electronic.

Subscriptions, requests for back issues and changes of subscribers address should be sent to Pacific Journal of Mathematics, P.O. Box 4163, Berkeley, CA 94704-0163, U.S.A. The Pacific Journal of Mathematics is indexed by Mathematical Reviews, Zentralblatt MATH, PASCAL CNRS Index, Referativnyi Zhurnal, Current Mathematical Publications and the Science Citation Index.

The Pacific Journal of Mathematics (ISSN 0030-8730) at the University of California, c/o Department of Mathematics, 798 Evans Hall \#3840, Berkeley, CA 94720-3840, is published monthly except July and August. Periodical rate postage paid at Berkeley, CA 94704, and additional mailing offices. POSTMASTER: send address changes to Pacific Journal of Mathematics, P.O. Box 4163, Berkeley, CA 94704-0163.

PJM peer review and production are managed by EditFLOW ${ }^{\circledR}$ from Mathematical Sciences Publishers.

PUBLISHED BY

mathematical sciences publishers

nonprofit scientific publishing

http://msp.org/

(C) 2013 Mathematical Sciences Publishers 


\section{PACIFIC JOURNAL OF MATHEMATICS}

Volume $264 \quad$ No. $1 \quad$ July 2013

On the center of fusion categories

ALAIN BRUGUIÈRES and ALEXIS VIRELIZIER

Connected quandles associated with pointed abelian groups

W. EdWin Clark, MoHamed ElHamdadi, Xiang-DONG HoU,

MASAHICO SAITO and TIMOTHY YEATMAN

Entropy and lowest eigenvalue on evolving manifolds

HongXin GuO, ROBERT PHILIPOWSKI and ANTON THALMAIER

Poles of certain residual Eisenstein series of classical groups

DIHUA JIANG, BAIYING LIU and LEI ZHANG

Harmonic maps on domains with piecewise Lipschitz continuous metrics 125

HAIGANG LI and CHANGYOU WANG

$q$-hypergeometric double sums as mock theta functions

JEREMY LOVEJOY and ROBERT OSBURN

Monic representations and Gorenstein-projective modules

XIU-HuA LUO and PU ZHANG

Helicoidal flat surfaces in hyperbolic 3-space

Antonio Martínez, JoÃo Paulo dos Santos and Keti

TENENBLAT

On a Galois connection between the subfield lattice and the multiplicative subgroup lattice

JOHN K. MCVEY

Some characterizations of Campanato spaces via commutators on Morrey 221 spaces

Shaoguang Shi and Shanzhen Lu

The Siegel-Weil formula for unitary groups 